Mon. Not. R. Astron. Soc. 000, 1 32 (2011)

Printed 21 June 2018

(MN LATEX style file v2.2)

\title{
Nature and chemical abundances of a sample of Lyman- $\alpha$ emitter objects at high redshift
}

\author{
O. L. Dors ${ }^{1 \star}$, B. Agarwal ${ }^{2}$, G. F. Hägele ${ }^{3,4}$, M. V. Cardaci ${ }^{3,4}$,Claes-Erik Rydberg $^{2}$, \\ R. A. Riffel ${ }^{5}$, A. S. Oliveira ${ }^{1}$, A. C. Krabbe ${ }^{1}$ \\ 1 Universidade do Vale do Paraíba, Av. Shishima Hifumi, 2911, Cep 12244-000, São José dos Campos, SP, Brazil \\ 2 Universität Heidelberg, Zentrum für Astronomie, Institut für Theoretische Astrophysik, Albert-Ueberle-Str. 2, D-69120 Heidelberg \\ 3 Instituto de Astrofísica de La Plata (CONICET-UNLP), Argentina. \\ ${ }^{4}$ Facultad de Ciencias Astronómicas y Geofísicas, Universidad Nacional de La Plata, Paseo del Bosque s/n, 1900 La Plata, Argentina. \\ 5 Universidade Federal de Santa Maria, Av. Roraima 1000, Cep 97105-900, Santa Maria, Brazil
}

Accepted 2015 Month 00. Received 2015 Month 00; in original form 2014 December 17

\begin{abstract}
We built a grid of photoionization models and compiled already available observational emission line intensities $(1000<\lambda(\AA)<2000)$ of confirmed star formation regions and Active Galactic Nucleus (AGNs) in order to classify five Ly $\alpha$ emitter (LAE) objects at high redshift $(5.7<z<7.2$ ). We selected objects for which at least one metal emission-line was measured. The resulting sample is composed by the objects RXC J2248.7-4431ID3, HSC J233408+004403, COSY, A1703-zd6, and CR7 (clump C). The photoionization models were built assuming a Power Law (associated with the presence of an AGN), a Direct Collapse Black Hole (DCBH), and Population II stars for the ionizing source. The resulting models were then compared with observational emission-line ratios in six diagnostic diagrams to produce a spectral classification of the sample. We found that CR7 (clump C), HSC J233408+004403 and COSY probably have a non thermal ionizing source (AGN or DCBH) while the RXC J2248.7-4431-ID3 and A1703-zd6 seem to host a stellar cluster. Detailed photoionization models were constructed to reproduce observational emission line ratios of the sample of LAEs, and to derive chemical abundances and number of ionizing photons $Q(\mathrm{H})$ of these objects. From these models, we found metallicities in the range $0.1 \lesssim\left(Z / Z_{\odot}\right) \lesssim 0.5$ and $\log Q(\mathrm{H})>53$. Values for $\mathrm{C} / \mathrm{O}$ abundance ratio derived for the LAEs
\end{abstract}


seem to be consistent with those derived for local star forming objects with similar metallicities, while an overabundance of N/O was found for most of the LAEs.

Key words: galaxies: active - galaxies: abundances - galaxies: evolution galaxies: nuclei - galaxies: formation- galaxies: ISM - galaxies: Seyfert

\section{INTRODUCTION}

The study of Lyman- $\alpha$ emitter objects (hereafter LAEs) at high redshift $(z>5)$ plays a key role in the understanding the reionization epoch of the Universe, chemical evolution and galaxy formation.

In their seminal work, Partridge \& Peebles (1967) predicted the existence of newly formed and highly luminous $\left(\sim 3 \times 10^{46} \mathrm{erg} / \mathrm{s}\right)$ galaxies present in an epoch when the Universe was 150 million years old (see also Pritchet 1994). Owing to the advancement of large ground-based telescopes (e.g. SUBARU; Very Large Telescope-VLT) together with the large amount of data produced by the Hubble Space Telescope (HST), several observational works have revealed the existence of these primeval galaxies, commonly referred to as LAEs (see e.g. Ouchi et al. 2008, 2009; Ota et al. 2008; Zheng et al. 2009; Lehnert et al. 2010; Stark et al. 2010; Zabl et al. 2015; Sobral et al. 2015; Oesch et al. 2015; Rvdberg et al. 2015, 2017; Schmidt et al. 2017; Mainali et al. 2017; Shibuva et al. 2018; Laporte et al. 2017, among others). In particular, spectroscopic data of LAEs have shown that these objects, in fact, exhibit a strong Ly $\alpha$ emission line $[\mathrm{L}(\mathrm{Ly} \alpha)]$ but show a weak emission of HeII $\lambda 1640 \AA$. Additionally, some objects exhibit metal emission lines in their spectra such as Nv $\lambda 1239 \AA$, CIV $\lambda 1550 \AA$ and OIII] $\lambda 1666 \AA$ (see Matthee et al. 2017; Shibuya et al. 2018).

Emission-line intensities and their ratios can be used to investigate the nature of the gas emission, i. e., to classify an object as a Star Forming region (SF) or an Active Galactic Nucleus (AGN). This methodology, based on optical emission lines, was proposed by Baldwin et al. (1981) 1 and hinges on the idea that the gas in AGNs presents a higher excitation degree and metallicity than the gas in SFs. Alternative diagnostic diagrams based on emission-line ratios have been proposed, e.g. in the near-infrared (IR) (Reunanen et al.

\footnotetext{
* 5 mail:olidors Quminap.br

1 Baldwin et al. (1981) considered four classes of objects: normal HiI regions, planetary nebulae, AGNs, and objects excited by shock-wave heating.
} 
2002; Rodríguez-Ardila et al. 2004; Riffel et al. 2013; Colina et al. 2015) and in the ultraviolet (UV; Allen et al. 1998; Feltre et al. 2016; Nakajima et al. 2017).

Studies based on diagnostic diagrams comprising of emission lines and on photometric data of LAEs have shown that there is no consensus on the nature of these objects. For example, using the X-SHOOTER and SINFONI instruments mounted on the VLT, DEIMOS on the Keck telescope, the near-IR COSMOS/UltraVISTA survey, and the WFPC3 on board the HST, Sobral et al. (2015) obtained spectroscopic and photometric data in the UV restframe of CR7 $(z=6.604)$. Using the high spatial resolution of HST data, they showed that CR7 is composed of three different spatially resolved components, namely A, B and C. They detected a strong Ly $\alpha$ and HeII $\lambda 1640 \AA$ emission, but their spectra did not show metal emission lines. Moreover, using this data together with IRAC/Spitzer mid-IR photometric data (optical data in the CR7 restframe) they concluded that a combination of population III stars which dominate the rest-frame UV light and the nebular emission lines, and a more metal enriched stellar population that dominates the mass, is the best explanation for the composition of CR7. However, Bowler et al. (2017), using almost the same data as Sobral et al. (2015) together with deeper IR and optical photometric data, arrived at a very different conclusion. They claimed that a low-mass narrow-line AGN or a young low metallicity stellar cluster with super solar $\alpha$-element abundance could be the ionizing source in CR7. On the other hand, Agarwal et al. (2017), by comparing the observed $H_{160^{-}}$[3.6] and [3.6]-[4.5] IR colors of CR7 obtained by Bowler et al. (2017) with photoionization model predictions, derived that the clump A potentially hosts an evolved direct collapse black hole (DCBH), while components $\mathrm{B}$ and $\mathrm{C}$ are consistent with metal enriched star forming regions (see also Pallottini et al. 2015; Pacucci et al. 2017). Recently, Sobral et al. (2017) presented new data for CR7 and concluded that this object seems to be actively forming stars, without any clear AGN activity in clumps A and B, with component A experiencing the most massive starburst. Their observations show that the component $\mathrm{C}$ hosts a strong ionization source, possibly an AGN (see also Visbal et al. 2016). Another disagreement is found, for example, for the LAE A1703-zd6 (at $z=7.043$ ). Stark et al. (2015) showed that photoionization models, considering both a non-thermal ionizing source (AGN) and stellar clusters can reproduce the observational data of this object, thus indicating a strong degeneracy of the results. They further argue that additional constraints on high ionization emission lines (e.g. NV $\lambda 1239 \AA$, CIV $\lambda 1550$ ) are needed in order to clarify the LAE's precise nature. 
The lack of HeII emission, the presence of narrow emission lines (with Full Width at Half Maximum (FWHM) between $\sim 100$ and $\sim 600 \mathrm{kms}^{-1}$ ) and the low Ly $\alpha$ equivalent widths $(\mathrm{EW}(\operatorname{Ly} \alpha) \lesssim 200 \AA)$ for most part of the LAEs (see Matthee et al. 2017; Shibuva et al. 2018; Leclerca et al. 2017) have been used as arguments favouring the low-metallicity stellar populations as the ionizing source of these objects (e.g. Mainali et al. 2017). However, the inability of SF in producing considerable Lyman continuum radiation into the intergalactic medium suggests that a significant contribution of ionizing radiation may emerge from AGNs (e.g. Madau \& Haardt 2015; Pallottini et al. 2015; Roberts-Borsani et al. 2016; Laporte et al. 2017). Moreover, Seyfert 2 type AGNs also show narrow-line profiles $(50 \lesssim$ FWHM $\lesssim 1200$, e.g Koski 1978; Bergeron et al. 1981; Dopita et al. 2015) and low equivalent widths $(\mathrm{EW}(\mathrm{Ly} \alpha) \lesssim 300 \AA$, e.g. Bergeron et al. 1981; Thuan 1984; De Robertis et al. 1988), indicating that LAEs could host some kind of non-thermal ionizing source. In summary, the exact nature of the LAEs still eludes us and remains an open challenge.

With the above in mind, in this paper we compare observational spectroscopic data of five LAEs, taken from the literature, with results of photoionization models generated by considering distinct types of ionizing sources. Our main goals are:

(i) Classifying LAEs at redshift $z>5$.

(ii) Determining the physical parameters that can best describe LAEs (e.g. metallicity, number of ionizing photons).

This paper is organized as follows. In Section 2, we present the observational data used in this study and in Section 3, we describe our photoionization models. In Section 4, the resulting classification and physical parameters that can help ascertain the nature of LAEs is presented. Finally, the discussion and conclusions are outlined in Sections 5 and 6 respectively.

\section{OBSERVATIONAL DATA}

In what follows, we describe the LAE data and the control sample. We compiled UV emissionline fluxes $(1000<\lambda(\AA)<2000)$ for a sample of LAEs located at redshift $z>5$. Also, with the goal of creating a control sample, UV emission-line fluxes of confirmed SFs, Seyfert 2 nuclei, Quasars and Radio Galaxies were also gathered from the literature. 


\section{$2.1 \quad$ LAEs}

Our LAE selection criteria was as follows:

(i) each selected object must be at $z>5$,

(ii) the flux of the Ly $\alpha \lambda 1216 \AA$ emission-line was measured,

(iii) the flux of HeII $\lambda 1640 \AA$ was measured or at least an upper limit was estimated,

(iv) the flux of at least one metal emission line of Nv $\lambda 1239 \AA$, CIv $\lambda 1550 \AA$, OIII $] \lambda 1666 \AA$, or CIII] $\lambda 1909 \AA$ was measured and, at least, one upper limit of the flux of one metal emission-line was estimated.

Throughout the paper, Civ $\lambda 1550 \AA$ refers to the sum of CIV $\lambda 1548.18 \AA$ and CIV $\lambda 1550.77 \AA$; $\operatorname{Nv} \lambda 1240 \AA$ is the sum of $\operatorname{Nv} \lambda 1238.8 \AA$ and $\operatorname{Nv} \lambda 1242.78 \AA$; and OIII] $\lambda 1666 \AA$ is the sum of OIII] $\lambda 1660.81 \AA$ and OIII] $\lambda 1666.15 \AA$. The selection criteria described above were employed so as to include each object in at least one diagnostic diagram involving metal emission lines.

The selection resulted in five objects, namely RXC J2248.7-4431-ID3 (hereafter ID3), CR7 (clump C), HSC J233408+004403 (hereafter J233408), COSY, and A1703-zd6. In Table 1 we summarise the redshift, emission-line fluxes, and the bibliographic reference for each object in our sample. In many cases, the study from which the data were extracted present only the upper limit estimates of the flux for some emission lines. This procedure is adopted by the authors because, often, a given line is not detected above $\sim 3 \sigma$ (e.g. CIV $\lambda 1550 \AA$ in CR7; Sobral et al. 2017). In particular, for A1703-zd6, only the OIII] $\lambda 1660.81 \AA$ line of the doublet was measured by (Stark et al. 2015). Thus, for this case, we adopted the theoretical $\operatorname{OiII}](\lambda 1660.81 \AA / \lambda 1666.15 \AA)=0.34$ line flux ratio to calculate the flux of OIII] $\lambda 1666 \AA$. In what follows, we summarize the results obtained in previous works for the considered LAEs.

\subsubsection{ID3}

Schmidt et al. (2017) used the Hubble Grism Lens-Amplified Survey from Space (GLASS), to obtain photometric and spectroscopic data of components of the system RXC J2248.74431 ( $z=6.11$ ), where the CIV emission was detected at 3-5 $\sigma$ level for two components. They compared the constraints of emission line flux ratios of the components of this galaxy with photoionization models and found that the observational data are better reproduced by SF rather than AGN models. Moreover, based on Spectral Energy Distribution (SED) fits, the authors found that the SF responsible for the ionization of the components must have a mass of $\sim 10^{9} M_{\odot}$, a Star Formation Rate (SFR) of $\sim 10 M_{\odot} /$ yr and an age younger than 50 Myr. 
By using the Folded-port InfraRed Echellette instrument coupled in the Magellan Baade

Telescope, Mainali et al. (2017) observed one component of RXCJ2248.7-443 identified by ID3. These authors were able to measure Ly $\alpha$ and some UV metal emission-line fluxes, however, no HeII flux was detected (see Table 1). They further concluded that the hard spectrum of this object is characteristic of low-metallicity stellar populations and is less consistent with AGN excitation.

\subsection{2 $C R 7$}

CR7 is one of the most luminous LAEs and was discovered by Sobral et al. (2015) using the data obtained with the Subaru Telescope by Matthee et al. (2015). Follow-up observations of CR7 using the F110W (YJ) and F160W (H) wide filters of the WFPC3 instrument on board the HST by Sobral et al. (2015), revealed that CR7 is composed of three components, namely A, B and C. Most recently, Sobral et al. (2017) presented new Hubble/WFC3 grism observations and a re-analysis of the VLT data of CR7, making it possible to obtain line fluxes of the components A, B and C. In their analysis, Sobral et al. (2017) measured the line NIV] $\lambda 1483.4 \AA, \lambda 1486.6 \AA$ in clump A (not considered in our analysis) and NV $\lambda 1240 \AA$ in clump C. Although CR7 is the most studied among the objects in our sample, its nature is still uncertain (see Sect. 1).

\section{$2.1 .3 \quad J 233408$}

Shibuya et al. (2018), using the Subaru Hyper Suprime-Cam (HSC) survey data, measured the Ly $\alpha$ and Civ fluxes and defined upper limits for Nv, HeII and OIII] lines of this object. These authors compared the HeII/CIV and OIII]/CIV ratios of this object with those observed in a sample of SFs and AGNs as well as with predictions of photoionization models built by Feltre et al. (2016). They found that the constraints on these ratios are more compatible with SFs rather than AGNs.

\subsubsection{COSY}

Laporte et al. (2017) used the XSHOOTER instrument on the VLT and the MOSFIRE instrument on the Keck telescope and obtained spectroscopic data of the LAE COSY (see also Stark et al. 2017). Laporte et al. (2017) measured the Ly $\alpha$, HeII and one metal line, i.e. Nv $\lambda 1240 \AA$ (see Table 1). From the comparisons of the ratios of the observed emission-line 
Table 1. Emission-line fluxes for LAEs compiled from the literature. Flux of Oin] $\lambda 1666$ for A1703-zd6 was calculated assuming the theoretical line flux ratio: OIII] $\lambda 1660.81 \AA / \lambda 1666.15 \AA=0.34$.

\begin{tabular}{|c|c|c|c|c|c|c|c|c|c|}
\hline Object & redshift & $\operatorname{Ly} \alpha \lambda 1216$ & Nv $\lambda 1239$ & Civ $\lambda 1549$ & HeII $\lambda 1640$ & Опіг] $\lambda 1666$ & С̆ін] $\lambda 1909$ & Flux $\left(\mathrm{erg} / \mathrm{s} / \mathrm{cm}^{2}\right)$ & Ref. \\
\hline ID3 & 6.110 & $33.2 \pm 2.3$ & $<1.8$ & $14.0 \pm 3.8$ & $<1.5$ & $4.4 \pm 0.85$ & $<3.6$ & $10^{-18}$ & 1 \\
\hline $\mathrm{CR} 7 \mathrm{C}$ & 6.604 & $2.6 \pm 1$ & $1.3 \pm 0.5$ & $<0.7$ & $1.0 \pm 0.4$ & $<0.8$ & $<1.0$ & $10^{-17}$ & 2 \\
\hline $\mathrm{J} 233408$ & 5.707 & $13.5 \pm 0.03$ & $<0.67$ & $1.15 \pm 0.14$ & $<0.16$ & $<0.09$ & - & $10^{-17}$ & 3 \\
\hline COSY & 7.149 & $22.9 \pm 3.0$ & $2.58 \pm 0.44$ & $<2.70$ & $1.26 \pm 0.29$ & - & $<1.75$ & $10^{-18}$ & 4 \\
\hline A1703-zd6 & 7.045 & $28.4 \pm 5.3$ & - & $7.9 \pm 1.13$ & $<2.1$ & 7.1 & - & $10^{-18}$ & 5 \\
\hline
\end{tabular}

fluxes CIII]/HeII, Nv/CIV, CIII]/HeII and Nv/HeII with those predicted by photoionization models built by Nakajima et al. (2017), Laporte et al. (2017) found that COSY is a likely AGN host.

\subsubsection{A1703-zd6}

A1703-zd6 is a bright LAE, identified by Bradley et al. (2012) for which first spectroscopic data was obtained by Schenker et al. (2012). Follow-up Keck/MOSFIRE observations by Stark et al. (2015) revealed the presence of UV metal emission-lines in the spectrum of this object. To interpret the combined stellar and nebular emission of this object, Stark et al. (2015) found that photoionization models can reproduce the observational data of A1703-zd6 if a young stellar population together with a gas component of metallicity $\left(Z / Z_{\odot}\right) \approx 0.02$, or an $\operatorname{AGN}$ with $\left(Z / Z_{\odot}\right)=0.001$ is assumed as the ionizing source.

\subsection{Control Sample}

We compiled observational UV narrow emission line intensities (FWHM $\lesssim 1000 \mathrm{~km} / \mathrm{s}$ ) of a sample of SFs and AGNs from the literature. Their emission-line intensity ratios were compared with those of the selected LAEs.

Concerning the SFs, we considered 13 objects at redshift $z<3.4$. This sample consists of 8 objects (Hir regions and starburst galaxies) compiled by Pérez-Montero \& Amorín (2017) whose data were obtained by Villar-Martín et al. (2004), Erb et al. (2010), Berg et al. (2016), de Barros et al. (2016), Steidel et al. (2016), and Vanzella et al. (2016). For the remaining five star forming galaxies, the data of one (object M0451) was obtained from Stark et al. (2014) and four (objects SB2, SB82, SB111, SB182) from Senchyna et al. (2017).

Regarding the AGN sample, we considered the observational data of 80 objects compiled by 
Dors et al. (2014), being 11 Seyfert 2 nuclei $(z<0.04), 10$ type 2 Quasars $(1.5<z<3.7)$ and 59 high- $z$ radio galaxies $(1.2<z<3.8)$. The reader is referred to Dors et al. (2014) for references pertaining to the original AGN sample.

\section{PHOTOIONIZATION MODELS}

We used the CLOUDY code version 17.00 (Ferland et al. 2013) to build a grid of photoionization models in order to compare the predicted UV emission-line intensities with those measured for the LAE spectra (see Sect. 2.1).

The models were built assuming a simple approach, i.e. a static spherical geometry with a central ionizing source, and constant electron density along the radius with different values of metallicity and number of ionizing photons emitted per second by the ionizing source. The gas ionization was assumed to be due to photoionization from radiation emitted by a unique source, and we did not consider gas shock ionization and heating. This is a good assumption for LAEs because the majority of these objects exhibit narrow emission lines, hinting that gas shock waves are absent, or have only a small influence on the ionization/heating of the gas. We assumed three distinct types for the ionizing sources: (i) a power law to represent Active Galactic Nuclei, (ii) Direct Collapse Black Hole and (iii) Population II stars. In what follows, the main parameters of the models are described.

\subsection{Active Galactic Nuclei}

To simulate the observational properties of AGNs, we considered the following values for the metallicity $(\mathrm{Z})$ in relation to the solar one $\left(Z_{\odot}\right):\left(Z / Z_{\odot}\right)=0.01,0.5,1.0$ and 2.0. These values cover the range of metallicities derived for AGNs located in a wide range of redshift $(0.0<$ $z<4.0$ ) as derived, for example, by Dors et al. (2014, 2015, 2017a), Castro et al. (2017), and Groves et al. (2006), who compared photoionization model results with observational narrow UV and optical emission line intensities of AGNs (see also Revalski et al. 2018; Thomas et al. 2018).

The abundance of the heavy elements was scaled with the oxygen abundance, with the exception of the nitrogen abundance, which was derived from the relation obtained by Dors et al. (2017a):

$\log (\mathrm{N} / \mathrm{H})=1.05( \pm 0.09) \times[\log (\mathrm{O} / \mathrm{H})]-0.35( \pm 0.33)$

The authors derived this relation using the CLOUDY code to build detailed photoionization 
models to reproduce observational optical narrow emission line intensities of 44 Sy2 AGNs at $z<0.1$ compiled from the literature.

For the models, we assumed an electron density value of $N_{\mathrm{e}}=500 \mathrm{~cm}^{-3}$, which is a representative value for the gas density of Narrow Line Regions as found by Dors et al. (2014). The SED was considered to be composed of two continuum components, where one represents the Big Blue Bump peaking at 1 Ryd, and the other is characterized by a power law with different values of the spectral index $\alpha_{o x}$ describing the continuum between $2 \mathrm{keV}$ and $2500 \AA$ (Zamorati et al. 1981). In the models, we consider the $\alpha_{o x}$ values $-0.8,-1.4$ and -2.0 , in order to cover the entire range of observed values derived by Miller et al. (2011). In Figure 1 we show the SEDs assuming these $\alpha_{o x}$ values, with logarithm of the number of ionizing photons emitted per second by the ionizing source $\log Q(\mathrm{H})=54$ and for $\lambda \leqslant 1000$ $\AA$.

The distance from the ionizing source to the innermost region of the gas $\left(R_{\text {int }}\right)$ was assumed to be $3 \mathrm{pc}$, which is similar to the radius derived in observational studies for narrow line regions (e.g. Jaffe et al. 2004; López-Gonzaga et al. 2016). The range of values for the number of ionizing photons was considered to be $50 \leqslant \log Q(\mathrm{H}) \leqslant 55$ with a step of 1 dex. In total, 90 photoionization models were built.

\subsection{Direct Collapse Black Hole}

For these models, the same AGN metallicity, $R_{\text {int }}, N_{\mathrm{e}}$ and $Q(\mathrm{H})$ values were assumed. The SEDs were considered to be multi-temperature black body spectra as defined by Shakura \& Sunvaev (1973), which simulate the radiation being emitted from a black hole accreting gas. We consider four distinct parameters for this system: black holes with masses of $10^{6} M_{\odot}$ and $10^{7} M_{\odot}$ accreting at $50 \%$ and $100 \%$ of the Eddington rate. These are the same SEDs considered in the photoionization models built by Agarwal et al. (2017).

The theory predicts that DCBHs form in an environment that is nearly metal-free in order to prevent fragmentation into stars (Bromm \& Loeb 2003; Omukai et al. 2008; Latif et al. 2016). However, the host haloes of these DCBHs quickly merge with a neighbouring galaxy and, therefore, the enriched gas in our models is a consequence of the later evolution of the DCBH, as proposed by Agarwal et al. (2013). In Fig. 2 the SEDs assumed for the DCBH models are presented. We can see that SED models with the same BH mass are very similar, implying that the mass accretion rate has a small influence on the SED. The model 


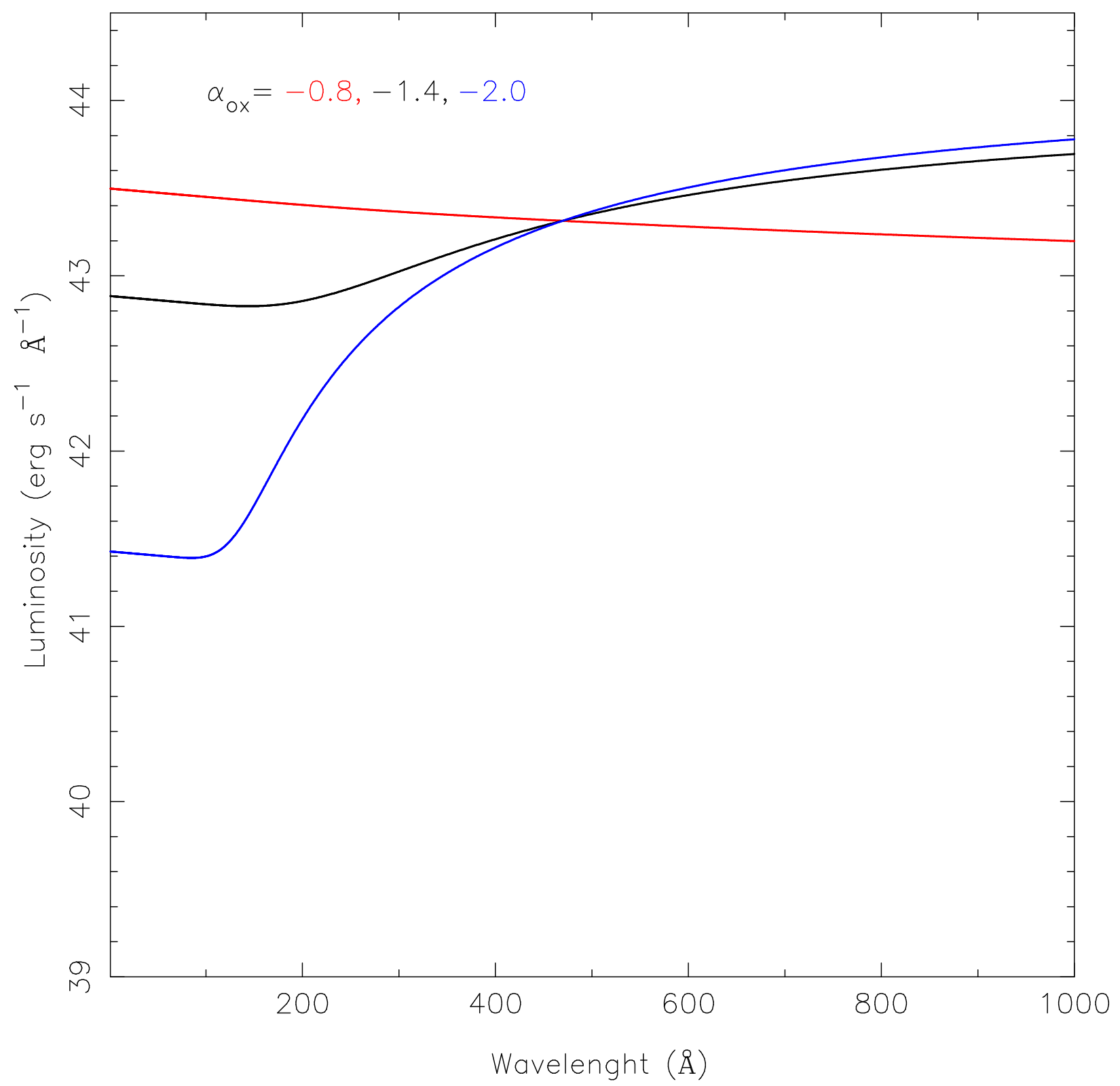

Figure 1. Rest-frame SED (logarithm of the luminosity vs. wavelength) in the UV spectral region $(\lambda \leqslant 1000 \AA)$ used in the AGNs photoionization models considering three $\alpha_{o x}$ values, represented with distinct colours, as indicated. The logarithm of the number of ionizing photons considered is $\log Q(\mathrm{H})=54$.

considering $10^{6} M_{\odot}$ is somewhat harder than the one for $10^{7} M_{\odot}$. A total of 95 models were built.

\subsection{Population II stars}

In the models for Population II (PopII) stars, the gas metallicity values were considered to be $\left(Z / Z_{\odot}\right)=0.03,0.2,0.5$, and 1.0 , about the same range of values found in HiI regions located in the disks of nearby spiral galaxies (e.g. Kennicutt et al.|2003; Díaz et al.|2007; Dors et al. 2017b) and in star-forming galaxies (e.g. Kewley \& Ellison 2008; Hägele et al. 


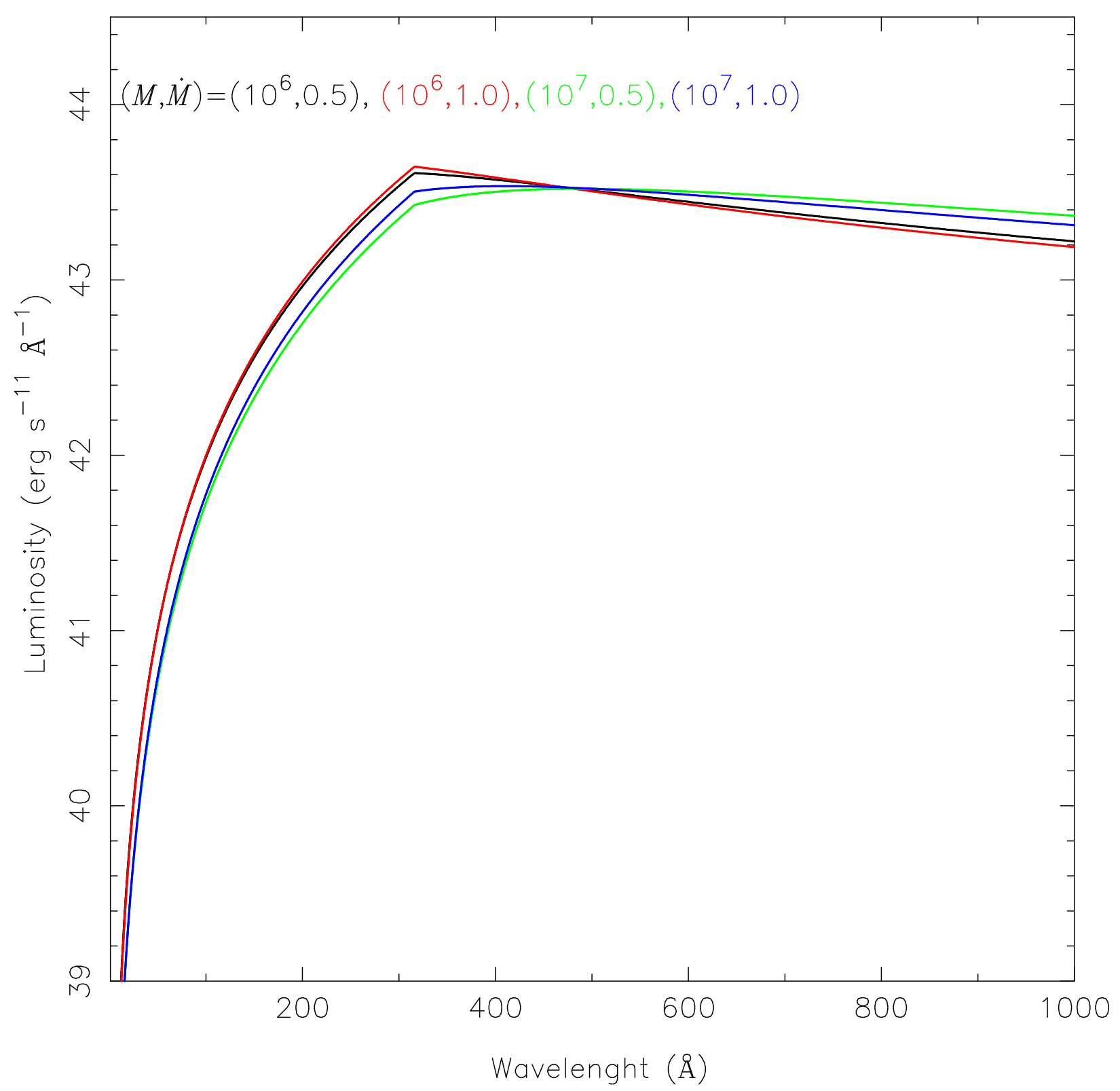

Figure 2. Similar to Fig. 1 but for the SEDs assumed in the DCBH models. Curves represent multi-temperature black body, whose radiation arises from a black hole with different masses and accreting gas at different rates, as indicated. The mass $M$ is in unit of solar masses and $\dot{M}$ is in units of Eddington rate.

2006, 2008, 2011, 2012). The electron density value was considered to be $N_{\mathrm{e}}=200 \mathrm{~cm}^{-3}$ in the models, which is in the range of those derived from the $[\mathrm{SII}] \lambda 6716 / \lambda 6731$ emission lines ratio for extragalactic HiI regions (Copetti et al. 2000; Krabbe et al. 2014; Sanders et al. 2016).

The abundances of heavy elements were linearly scaled with the oxygen abundance, with exception of $\mathrm{N}$ and $\mathrm{C}$. For nitrogen, we used the following relation between this element and oxygen derived by Vila-Costas \& Edmunds (1993):

$\log \mathrm{N} / \mathrm{H}=\log (\mathrm{O} / \mathrm{H})+\log (0.034+120 \times \mathrm{O} / \mathrm{H})$ 
Regarding carbon, there are hints suggesting that galaxies located at high redshift $(z=$ $2-4$ ) have an enhanced $\mathrm{C} / \mathrm{O}$ abundance ratio in comparison to the objects at low redshift (Nakajima et al. 2017). Since the C/O-O/H abundance relation assumed in the models affects the predicted strengths of CIII] and Civ lines (Nakajima et al. 2017), we consider two relations in our grid of models. Firstly, a grid of photoionization models was built considering $\log (\mathrm{C} / \mathrm{O})$ equal to the solar ratio $\log (\mathrm{C} / \mathrm{O})_{\odot}=-0.30$ obtained by Allende Prieto et al. (2001, 2002). Alternatively, another grid of models was built assuming the following derived by Dopita et al. (2006):

$\mathrm{C} / \mathrm{H}=6.0 \times 10^{-5} \times\left(\mathrm{Z} / \mathrm{Z}_{\odot}\right)+2.0 \times 10^{-4} \times\left(\mathrm{Z} / \mathrm{Z}_{\odot}\right)^{2}$.

As a first step, the ionizing source was considered to be a stellar cluster that formed instantaneously (i.e burst) with a mass of $10^{6} M_{\odot}$, following an Initial Mass Function (IMF) with exponents of 1.3 for stellar masses from 0.1 to $0.5 M_{\odot}$ and 2.3 for stellar masses between 0.5 and $100 M_{\odot}$. These parameters were assumed as our input for the STARBURST99 code (Leitherer et al. 1999) in order to generate the SEDs. Two stellar tracks were considered in the STARBURST99 models: (i) the GENEVA tracks with stellar rotation (Levesque et al. 2012) with metallicities of 0.7 and $1.0 Z / Z_{\odot}$ (the only available metallicities) and (ii) the PADOVA tracks with AGB stars (Bertelli et al. 1994) with metallicities of $0.02,0.2,0.4$, and $1.0 Z / Z_{\odot}$. Three ages were considered for the stellar cluster: $10^{4} \mathrm{yr}, 2.5 \mathrm{Myr}$, and 6.0 Myr. Theoretical studies based on comparison between emission-line intensities of HiI regions predicted by photoionization models and observational data have found ionizing stellar cluster with ages in this range (Copetti et al. 1985; Bresolin et al. 1999; Stasinska \& Izotov 2003; Dors \& Copetti 2006). We consider the WM-basic stellar atmosphere models of Paudrach et al. (2001), which seem to reproduce the best agreement between predicted and observed optical emission-line ratios of HiI regions (Zastrow et al. 2013). In the upper panel of Fig. 3, the GENEVA stellar cluster SEDs with different ages and the same metallicity $\left(\mathrm{Z} / \mathrm{Z}_{\odot}=1.0\right)$ are shown. In the bottom panel of the same figure, a comparison between the stellar clusters SEDs from GENEVA assuming an age of 0.01 Myr and two different metallicities $\left(\mathrm{Z} / \mathrm{Z}_{\odot}=1.0\right.$ and $\left.\mathrm{Z} / \mathrm{Z}_{\odot}=0.7\right)$ are shown. The dependence of the SED on both the age and metallicity can be seen in the figure (see also Senchyna et al. 2017; Leitherer et al. 1999).

Thus, we obtained 18 different SED models with $\log Q(\mathrm{H})$ varying between $\sim 51.1$ and $\sim 52.8$. In the second step we built the complete PopII photoionization models by varying the 


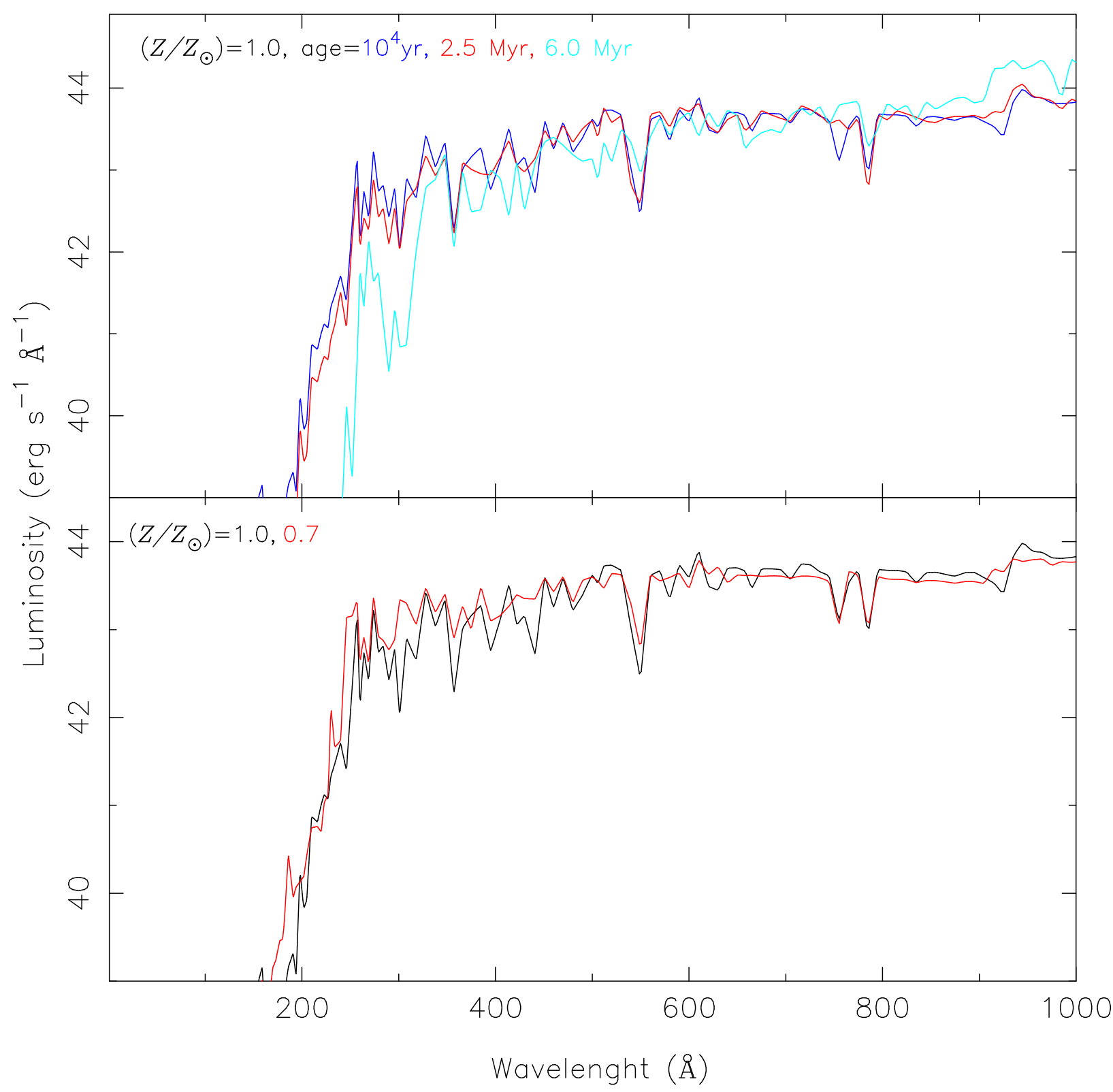

Figure 3. Rest-frame SEDs of PopII stellar clusters built using the STARBURST99 code (Leitherer et al. 1999) and the GENEVA stellar tracks with rotation (Levesque et al. 2012). In the upper panel we compared SEDs with $\mathrm{Z} / \mathrm{Z}_{\odot}=1.0$ and different ages as labelled. In the lower panel we show a comparison between SEDs built assuming an age of $0.01 \mathrm{Myr}$ and two different metallicities. In all cases the WM-basic stellar atmospheres (Paudrach et al. 2001) were assumed.

logarithm of the number of ionizing photons in the range $49 \leqslant \log \mathrm{Q}(\mathrm{H}) \leqslant 56$, with a step of 1 dex. Since the number of ionizing photons is directly proportional to the stellar cluster mass, this $Q(\mathrm{H})$ range, considering the STARBURST99 (Leitherer et al. 1999) predictions, can be obtained by varying the stellar cluster mass in the range $\sim 10^{4}$ to $\sim 10^{11} M_{\odot}$. In order to build realistic models, the metallicity of the gas phase was matched with the closest available metallicity of the stellar atmospheres (see Dors et al. 2011 for a discussion about this methodology). In total, 272 models were built.

In Fig. 4 we present a comparison between the hardest SED of each type, as described 


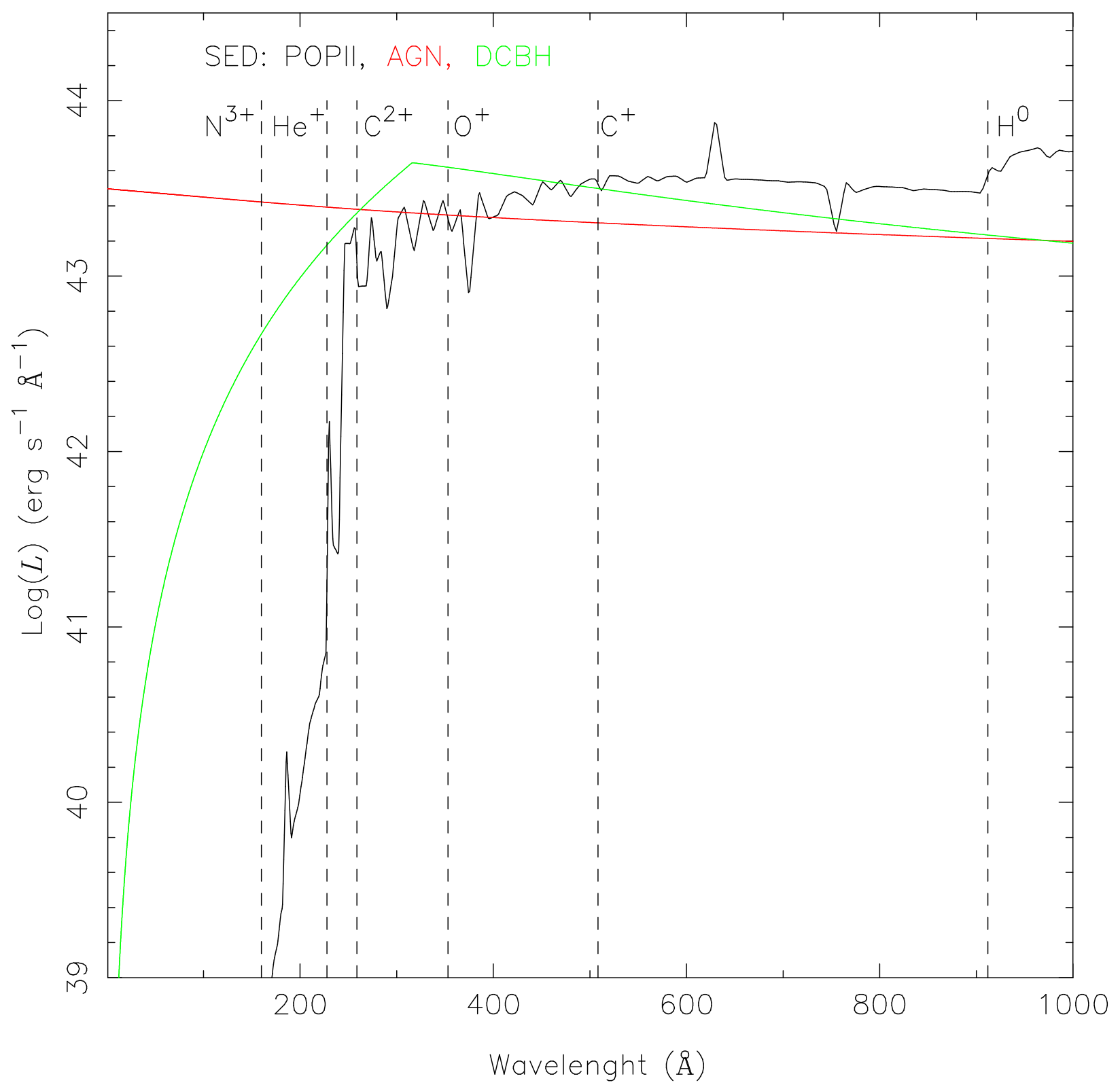

Figure 4. Comparison among the hardest SED of each type considered in the models (as indicated) and described in Sect. 3 Dashed lines mark the wavelengths corresponding to the ionization potential of $\mathrm{H}^{0}, \mathrm{C}^{+}, \mathrm{O}^{+}, \mathrm{C}^{2+}, \mathrm{He}^{+}$and $\mathrm{N}^{3+}$. The hardest SED for PopII stars corresponds to the one with the lowest metallicity $\left(Z / Z_{\odot}=0.02\right)$ and 0.01 Myr old, for AGNs it corresponds to the one with $\alpha_{o x}=-0.8$ and for DCBH it corresponds to the one with a mass of $10^{6} M_{\odot}$ accreting $100 \%$ at Eddington rate.

previously. It shows that our AGN model exhibits the hardest SED, followed by the DCBH and PopII model.

\section{RESULTS}

\subsection{Spectral Classification}

In order to spectroscopically classify the five selected LAEs, we took into account diagnostic diagrams containing observational line ratios of the control sample and the ones predicted 
Table 2. Indication of which type of model grid (see Section 3) reproduces the observed emission line ratios of the sample of objects in the diagrams presented in Fig. 8 Between parentheses, the alternative classification that could be derived if we take into account the arrows (added when only upper or lower limits of the line ratios are available) and the intrinsic errors in the line-curves determinations are indicated.

\begin{tabular}{|c|c|c|c|c|c|}
\hline & \multicolumn{5}{|c|}{ Classification } \\
\hline & ID3 & $\mathrm{CR} 7(\mathrm{C})$ & $\mathrm{J} 233408$ & COSY & A1703-zd6 \\
\hline \multicolumn{6}{|l|}{ Diagram } \\
\hline & \multicolumn{5}{|c|}{ Empirical } \\
\hline CiII]/HeII vs. Nv/HeII & - & AGN & - & AGN & - \\
\hline CIII]/HeII vs. Civ/ HeII & - & $\mathrm{AGN}$ & - & AGN & - \\
\hline C43 vs. CinI]/Civ & SF & - & - & AGN & - \\
\hline СıII]/HeII vs. OІІІ]/HeII & - & $\mathrm{SF}(\mathrm{AGN})$ & - & - & - \\
\hline \multirow[t]{2}{*}{ CIv/Ly $\alpha$ vs.Oiı]/Ly $\alpha$} & AGN (SF) & $\mathrm{SF}(\mathrm{AGN})$ & AGN & - & $\mathrm{SF}$ \\
\hline & \multicolumn{5}{|c|}{ Theoretical } \\
\hline CiII]/HeII vs. Nv/HeII & - & AGN & - & AGN & - \\
\hline CIII]/HeII vs. Civ/ HeII & - & AGN & - & AGN & - \\
\hline C43 vs. Ciri]/Civ & AGN (SF) & - & - & AGN & - \\
\hline CIII]/HeII vs. OIII]/HeII & - & AGN & - & - & - \\
\hline Civ/Ly $\alpha$ vs.Oiı] /Ly $\alpha$ & $\mathrm{SF}(\mathrm{AGN})$ & $\mathrm{SF}(\mathrm{AGN})$ & AGN & - & SF \\
\hline Adopted classification & SF & AGN & $\mathrm{AGN}$ & AGN & $\mathrm{SF}$ \\
\hline
\end{tabular}

by the photoionization models, and created demarcations between the regions occupied by SFs and AGNs.

In Fig. 5, bottom panel, we compared the logarithm of the luminosity of Ly $\alpha$ and HeII calculated for the LAEs with the ones of the control sample. The luminosity of each object was calculated using the fluxes listed in Table1 1 and the fluxes obtained from the papers from which the control sample data were compiled. The distance to each object was calculated using its redshift and assuming a spatially flat cosmology with $H_{0}=71 \mathrm{~km} \mathrm{~s}^{-1} \mathrm{Mpc}^{-1}, \Omega_{m}=$ 0.270, and $\Omega_{\mathrm{vac}}=0.730$ (Wright 2006). Unfortunately, in Fig. 5, it was possible to calculate the Ly $\alpha$ and HeI luminosities only for three SFs (the objects observed by de Barros et al. 2016; Steidel et al. 2016; Erb et al. 2010). From this plot, one can conclude the following:

(i) A trend is apparent.

(ii) LAEs show luminosities similar to those seen for Quasars and less bright Radio Galaxies.

(iii) The Ly $\alpha$ luminosities of the LAEs are spread in a range of about a factor of 5 , while the HeII ones present variations of about an order of magnitude, being relatively concentrated if we compared their luminosities with those of the objects in the control sample.

(iv) LAE luminosities are higher than those of Seyfert 2 nuclei (except for the most luminous Seyfert 2 object).

(v) More data for SF is necessary to improve the comparison between 


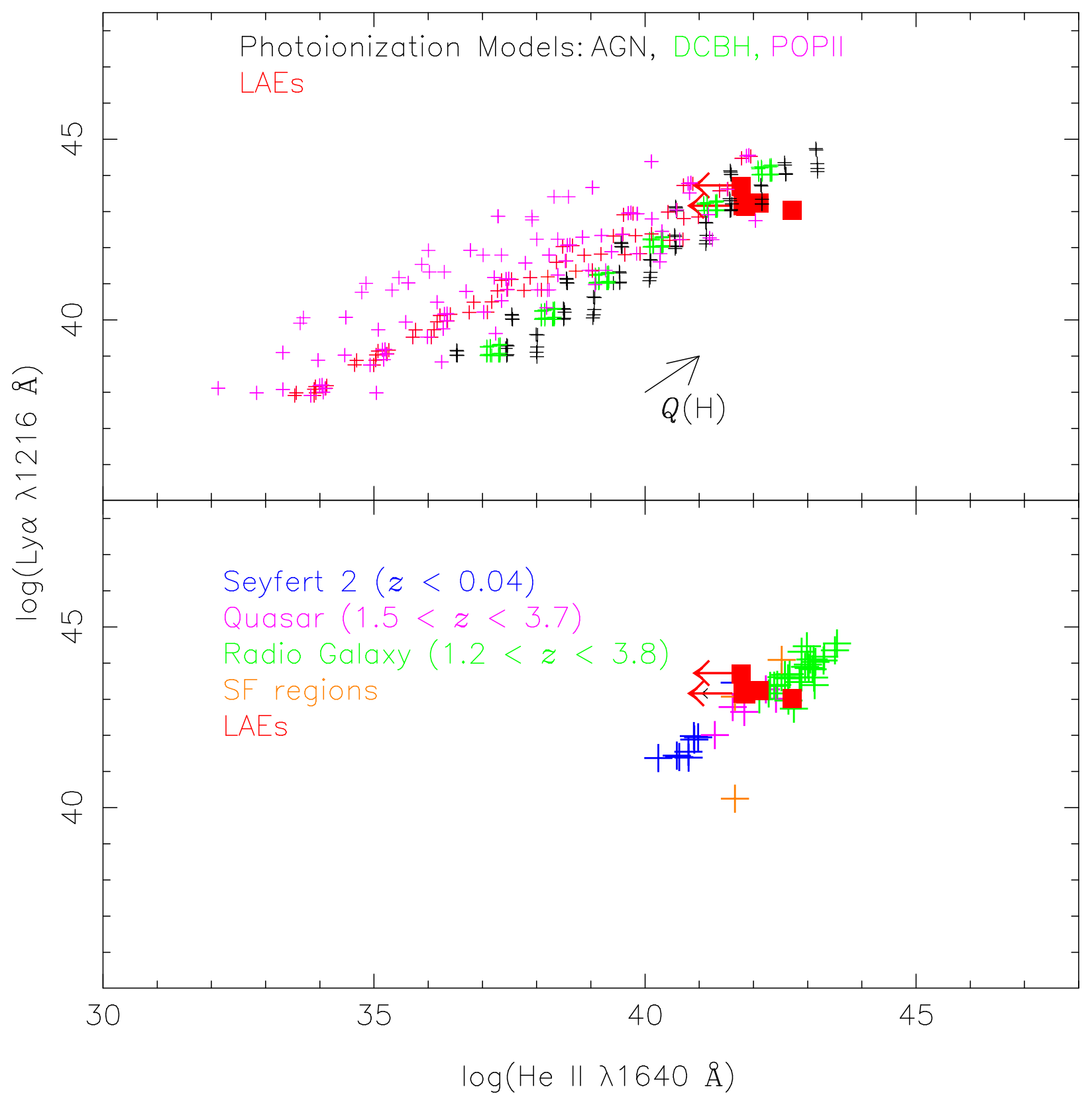

Figure 5. Logarithm of the Ly $\alpha$ luminosity versus the logarithm of HeII $\lambda 1640 \AA$ luminosity. Bottom panel: Comparison between the luminosities of the objects of the control sample (see Section 2.2) and those of the LAEs listed in Table 1 Upper panel: Comparison between the luminosities predicted by the models (see Sect. 3) and of the LAEs. The black arrow indicates the direction in which the number of ionizing photons increases in the models. The meaning of each symbol is indicated in the plots. The arrows linked to the object symbols indicate that only the upper limit of the HeII luminosity values were quoted. Typical errors in the luminosity values are about 0.1 dex (see e.g. Matthee et al. 2017; Shibuva et al. 2018).

these objects and LAEs.

In the upper panel of Fig. 5 we compare the LAE luminosities with those predicted by our grid of photoionization models (see Sect. 3). We found that model results describe the LAE data well only when values of $Q(\mathrm{H})$ higher than 53 dex (value not indicated in the plot) are considered. Furthermore, no clear distinction among models built using different kind of ionizing sources can be made. 
In Fig. 6, six diagnostic diagrams considering different emission-line ratios for the control sample are shown. In the CIV/Ly $\alpha$ versus Nv/Ly $\alpha$ diagram (bottom-right panel) it was not possible to include SF due to lack of the involved emission-lines. Objects with only upper limits of the emission-line ratio are not included in the diagrams. Regardless of the limited SF data, we define demarcations between the zones occupied by SFs and AGNs in all diagrams, with the exception of the $\mathrm{CIV} / \mathrm{Ly} \alpha$ vs. Nv/Ly $\alpha$ diagram. It is worth mentioning that due to the fact the $\operatorname{Ly} \alpha$ is a resonant transition and is highly scattered by the neutral hydrogen in the Intergalactic Medium at high redshifts $(z>5)$, the Ly $\alpha$ flux is usually observed at about $5-10 \%$ of its intrinsic output. The effect of a Ly $\alpha$ attenuation in the CIV/Ly $\alpha$ vs. OIII]/Ly $\alpha$ diagram would result in these objects moving almost parallel to the curves that separate SF from AGNs, since Ly $\alpha$ is in the denominator of both the axes.

The same set of diagnostic diagrams are also built considering our photoionization model results, as shown in Fig. 7 (see also Feltre et al. 2016; Nakajima et al. 2017, 2018; Sobral et al. 2018). In these diagrams, we can see that AGN and DCBH models occupy the same region, that is clearly different from the region occupied by the SF models, with exception of the Crv/Ly $\alpha$ vs. Nv/Ly $\alpha$ diagnostic diagram where the different models overlap. Demarcations between the zones occupied by SFs and AGNs were also defined. These zones are different from the ones empirically found using the control sample.

Similar diagnostic diagrams as the ones in Figs. [6 and 7 are plotted in Fig. 8 for the LAE sample using the line fluxes compiled from the literature and listed in Table 1. In order to classify the LAEs using these diagnostic diagrams, we included zone-separation curves obtained using the control sample (empirical classification, see Fig. 6) and those from the diagrams containing photoionization models (theoretical classification, see Fig. 7). The Civ/Ly $\alpha$ vs. Nv/Ly $\alpha$ diagnostic diagram was not included in this figure since it does not provide a method to distinguish AGN-like from SF-like objects.

Table 2 lists the classification for each LAE based on each diagnostic diagram, and the resulting classification, i.e. the frequency of the highest occurrence of a given object class. We marked in Table 2, between parentheses, the alternative classification that could be derived if we take into account the arrows (added when only upper or lower limits of the line ratios are available) and the intrinsic errors in the line-curves determinations. Three of the LAEs in our sample (CR7 (C), J233408 and COSY) can be classified as AGN-like objects using our diagnostic diagrams and two of them, ID3 and A1703-zd6, can be classified as SF-like objects. 

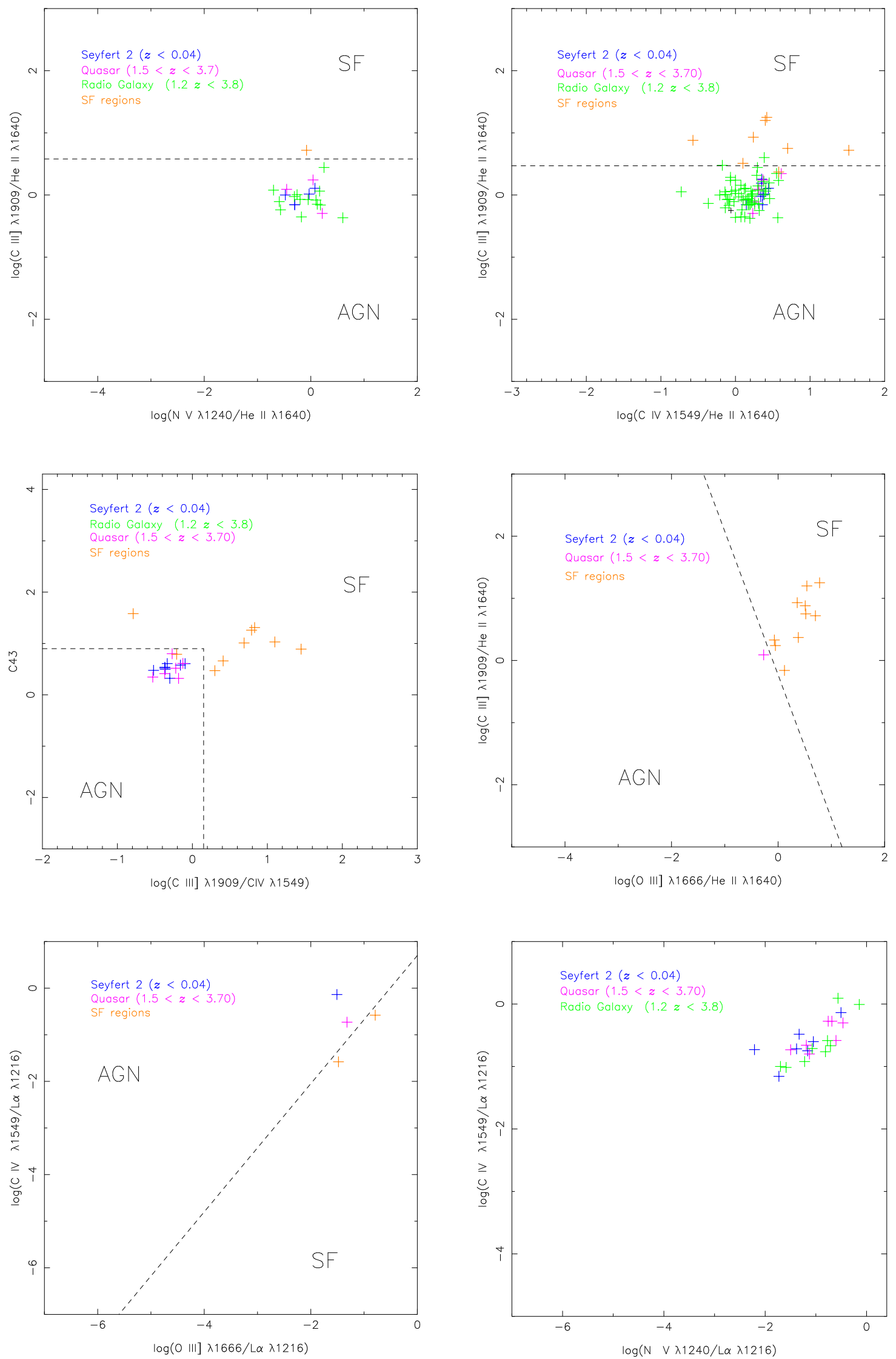

Figure 6. Diagnostic diagrams considering different emission-line ratios for the control sample (see Section 2.2). The C43 refers to $\log [(\operatorname{CrV} \lambda 1549+\mathrm{CIII}] \lambda 1909) /$ HeII $\lambda 1640]$ (Dors et al. 2014). The line curves separate SF-like objects zone from that of 

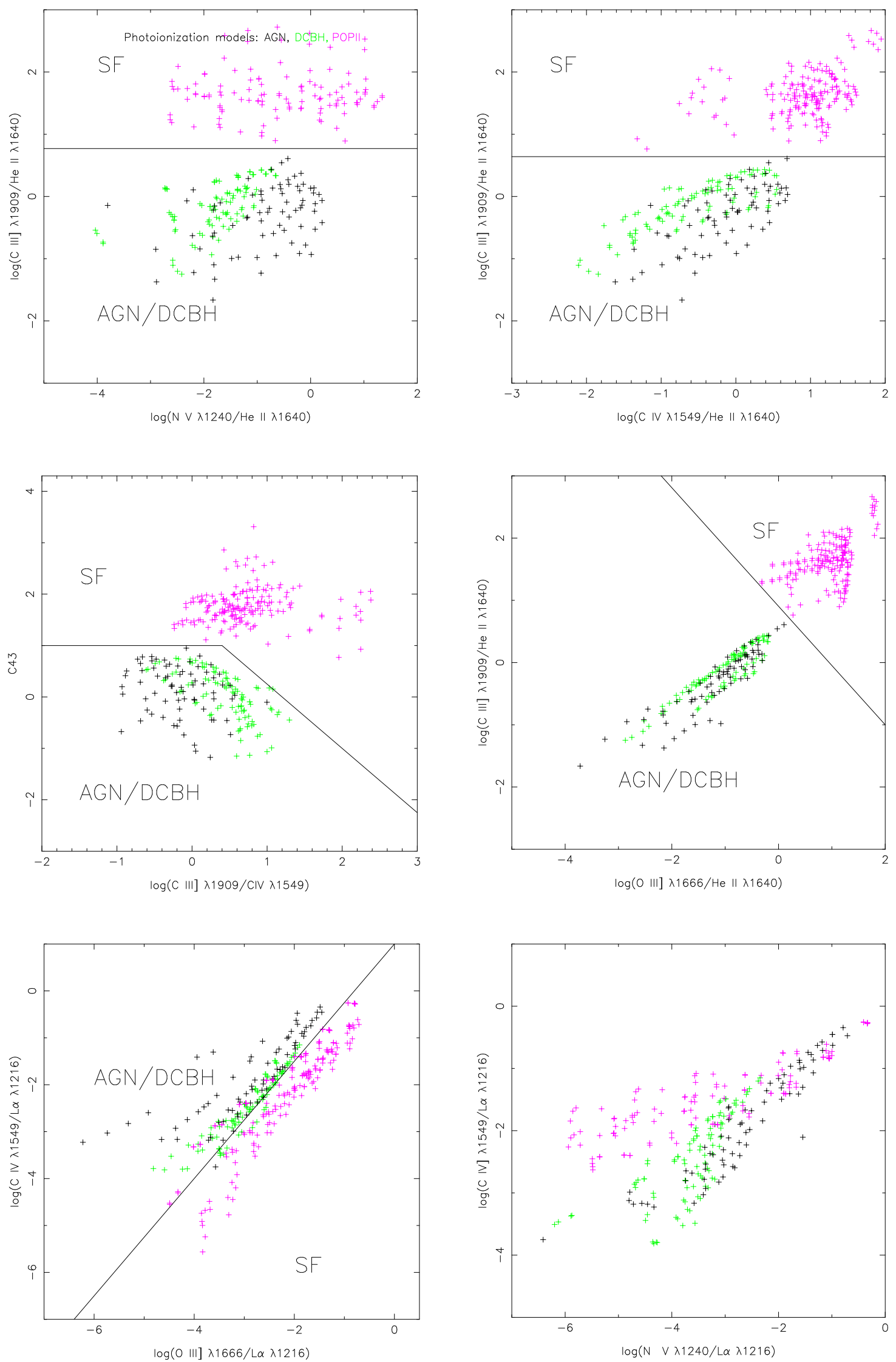

Figure 7. Same as Fig. 6 but considering line ratio intensities predicted by the photoionization models (see Sect. 3). Symbols with different colours represent models with distinct ionizing source as indicated. 

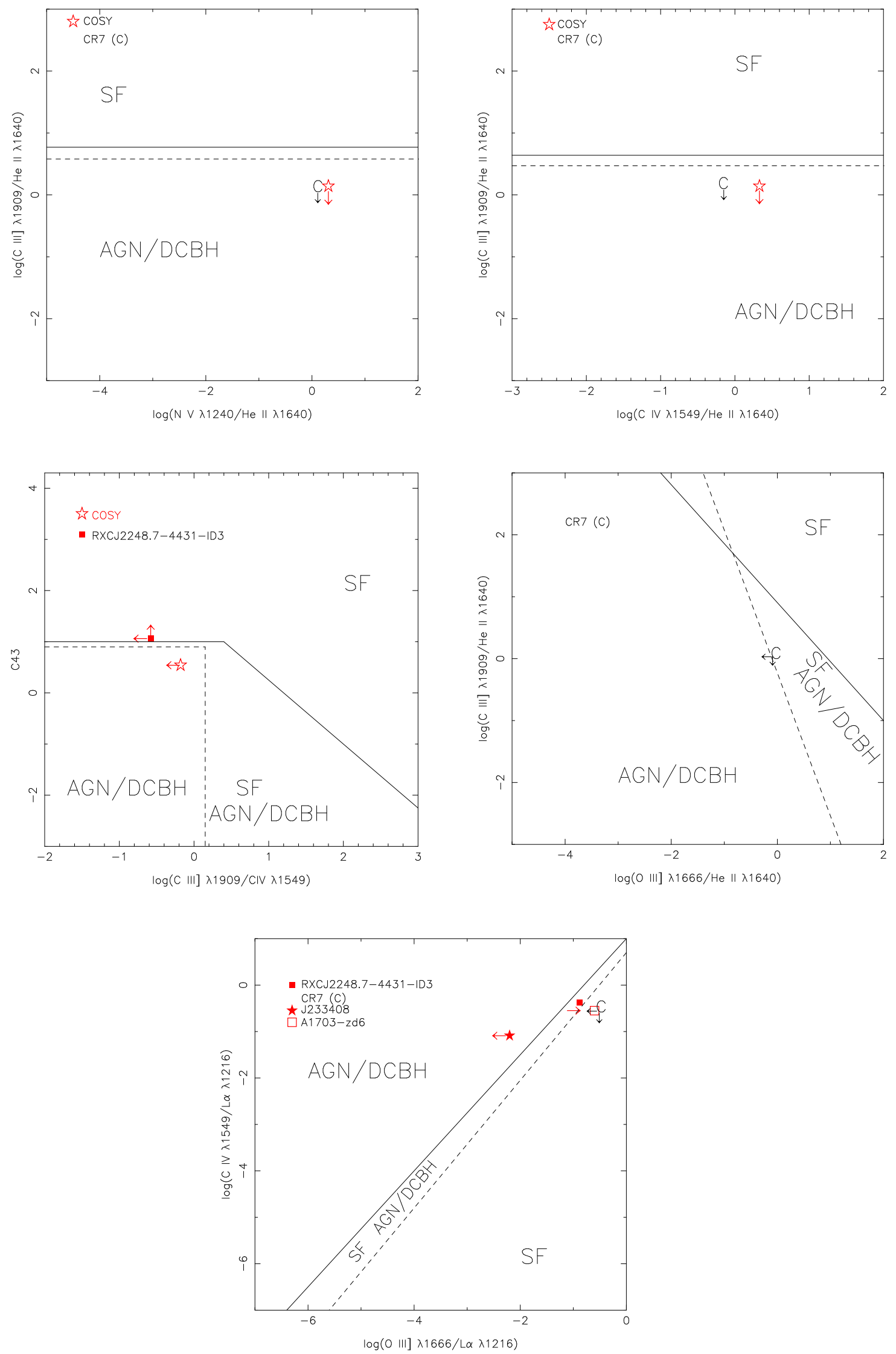

Figure 8. Diagnostic diagrams considering the emission line ratios of the sample of LAEs (see Sect. 2.1). Line curves separate SFs zone from that of AGNs as defined in Figs. 6 and 17 Each LAE is represented by different symbol as indicated. Arrows indicate that only the upper and/or lower limit value of a given emission-line ratio was quoted. 


\subsection{Detailed models}

Once the spectral classification of the LAEs is adopted (see Table 2), it is possible to derive some parameters describing them by building detailed photoionization models. The standard procedure is to reproduce a ratio between a given metal emission line and one hydrogen emission line used as reference (e.g. Dors et al. 2011, 2017a; Contini 2017). However, since in some cases the Lyman continuum can escape from a galaxy (e.g. Rivera-Thorsen et al. 2017) and due to the scattering of the Ly $\alpha$ photons by neutral hydrogen (e.g. Haves 2015), we used the intensities of metal lines in relation to the HeII $\lambda 1640 \AA$ as an observational constraint.

The methodology adopted is similar to that of by Dors et al. (2017a), who built detailed photoionization models to reproduce optical emission lines of Seyfert $2 \mathrm{~s}$ in order to derive their chemical abundances. An initial model assuming the following input parameters was built with:

(i) Metallicity - An initial value $Z / Z_{\odot}=0.1$ was assumed. This value is in the metallicity range derived, for example, for CR7 (Sobral et al. 2017; Agarwal et al. 2017) and for A1703zd6 (Stark et al. 2015).

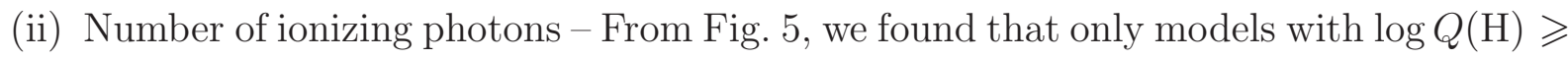
53 predict Ly $\alpha$ and HeII luminosity observed for the objects in our sample of LAEs. Thus, we adopted $\log Q(\mathrm{H})=53$ as an initial value for the number of ionizing photons.

(iii) SED - For each LAE, the ionizing source type was assumed to be the one derived in the adopted classification listed in Table 2, For ID3 and A1703-zd6, the objects in the sample classified as SF, we adopted the SED for a stellar cluster with an age of $10^{4} \mathrm{yr}$ (see Section 3.3). For the objects classified as AGN-like, we adopted both DCBH ( $M=$ $\left.10^{6} M_{\odot} ; \dot{M}=1.0\right)$ and power law $\left(\alpha_{\text {ox }}=-0.8\right)$ SEDs.

(iv) $\mathrm{N}$ and $\mathrm{C}$ abundances - The $\mathrm{N}$ and $\mathrm{C}$ abundances were calculated from Eqs. 2 and 3 , respectively.

(v) Electron density - The value of the electron density was considered to be $N_{\mathrm{e}}=$ $500 \mathrm{~cm}^{-3}$. The electron density in LAEs at high redshift is unknown and the value above only represents gas in the low density limit, where collisional de-excitation does not affect the line formation.

We then ran new models varying $Z, \mathrm{~N} / \mathrm{H}, \mathrm{C} / \mathrm{H}$ values separately in steps of $\pm 0.2 \mathrm{dex}$, which is the typical uncertainty in nebular abundance estimations derived through photoionization 
Table 3. Comparison between the logarithm of observational emission-lines ratios (calculated using the fluxes listed in Table 1 ) with the ones predicted by our detailed photoionization models. The observed values compiled from the literature are referred as "Obs." while the predicted values by the photoionization models as "Mod."

\begin{tabular}{|c|c|c|c|c|c|c|c|c|c|c|c|c|c|}
\hline & \multicolumn{3}{|c|}{ COSY } & \multicolumn{2}{|c|}{ ID3 } & \multicolumn{3}{|c|}{ CR7(C) } & \multicolumn{3}{|c|}{$\mathrm{J} 233408$} & \multicolumn{2}{|c|}{ A1703-zd6 } \\
\hline & Obs. & $\mathrm{AGN}^{\mathrm{N}}$ & d. & Obs. & $\begin{array}{c}\text { Mod. } \\
\text { SF }\end{array}$ & Obs. & $\mathrm{AGN}^{\mathrm{M}}$ & d. & Obs. & $\mathrm{AGN}^{\mathrm{l}}$ & $\begin{array}{l}\text { d. } \\
\text { DCBH }\end{array}$ & Obs. & $\begin{array}{l}\text { Mod. } \\
\text { SF }\end{array}$ \\
\hline$\frac{\operatorname{Ly} \alpha}{\text { HeII }}$ & $1.26 \pm 0.11$ & 1.29 & 1.75 & $>1.34$ & 2.88 & $0.41 \pm 0.21$ & 1.19 & 1.70 & $>1.92$ & 1.12 & 1.77 & $>1.13$ & 3.27 \\
\hline$\frac{\text { C III] }}{\text { HeII }}$ & $<0.03$ & -0.03 & -0.35 & - & - & $<-0.00$ & -0.69 & -0.44 & - & - & - & - & - \\
\hline$\frac{\mathrm{N} V}{\mathrm{He} I \mathrm{I}}$ & $0.30 \pm 0.12$ & 0.27 & 0.26 & - & - & $0.11 \pm 0.24$ & 0.14 & -0.01 & - & - & - & - & - \\
\hline$\frac{\mathrm{C} \mathrm{IV]}}{\text { HeII }}$ & $<0.33$ & 0.30 & 0.30 & $>0.97$ & 0.97 & $<-0.15$ & -0.19 & -0.22 & $>0.85$ & 0.83 & 0.88 & $>0.57$ & 0.57 \\
\hline$\frac{\mathrm{O} \text { III] }}{\text { HeII }}$ & - & - & - & $>0.46$ & 0.56 & $<-0.09$ & -0.24 & -0.12 & - & - & - & $>0.52$ & 0.85 \\
\hline
\end{tabular}

models (Dors et al. 2011). The $\log Q(\mathrm{H})$ was varied considering a step of 0.5 dex. Only one parameter was varied at a time and the optimization method PHYMIR (van Hoof 1997) was employed to select the best fitting model for the set of emission line intensities. For ID3, J233408 and A1703-zd6 the Nv line was not measured, therefore, the nitrogen abundance assumed in the models was scaled directly from the oxygen abundance by using Equation 2 .

In Table 3, the observed emission-line ratios and the ones predicted by our detailed models built to represent each object of the sample are listed. The best fit parameters of the models are listed in Table 4. The ionization parameter $U$, listed in Table 4 for each LAE, is not an input parameter of the models. It is defined as $U=Q(\mathrm{H}) / 4 \pi R_{\text {in }}^{2} n c$, where $Q(\mathrm{H})$ is the number of hydrogen ionizing photons emitted per second by the ionizing source, $R_{\text {in }}$ is the distance from the ionization source to the inner surface of the ionized gas cloud (in $\mathrm{cm}$ ), $n$ is the particle density (in $\mathrm{cm}^{-3}$ ), and $c$ is the speed of light.

To estimate the error in the parameters derived for each object, we adopted a similar methodology as the one employed by Dors et al. (2017a). Firstly, we consider the final model solutions for each LAE (see Table 4). From these models, we vary the metallicity (with a step of 0.05 dex), ionization parameter (step of $0.1 \mathrm{dex}$ ) and the nitrogen abundance (with a step of 0.05 dex) in order to obtain the range of parameter values for which the models reproduce the observational uncertainties of the emission line ratios of each LAE. This procedure was carried out only for COSY and CR7(C) because only these two objects have measurements of the HeII $\lambda 1640 \AA$ flux, making it possible to quote parameter values. However, carbon emission line fluxes were not measured in the spectrum of these objects, therefore, it was not possible to estimate the uncertainty in the $\mathrm{C}$ abundance estimations. For the other objects (ID3, J233408 and A1703-zd6), the HeII line flux was not constrained, therefore, the parameter values derived for them must be interpreted as upper limits. 
Table 4. Best fit nebular parameter used to reproduce the emission line ratio observed in the LAE sample. Results for a same object considering different ionizing sources (AGN, DCBH, SF) in the models are presented separately. The values of the parameters for ID3, J233408 and A1703-zd6 must be considered as upper limit values (see Sect. 4.2).

\begin{tabular}{|c|c|c|c|c|c|c|c|c|}
\hline \multirow[b]{2}{*}{ Parameter $\backslash \mathrm{SED}$} & \multicolumn{2}{|c|}{ COSY } & \multirow{2}{*}{$\begin{array}{l}\text { ID3 } \\
\text { SF }\end{array}$} & \multicolumn{2}{|c|}{ CR7(C) } & \multicolumn{2}{|c|}{ J233408 } & \multirow{2}{*}{$\begin{array}{c}\text { A1703-zd6 } \\
\text { SF }\end{array}$} \\
\hline & AGN & $\mathrm{DCBH}$ & & AGN & $\mathrm{DCBH}$ & AGN & $\mathrm{DCBH}$ & \\
\hline $\log Q(\mathrm{H})$ & $53.45_{-0.30}^{+0.75}$ & $56.0_{-1.10}^{+0.50}$ & 55.98 & $56.0_{-2.8}$ & $55.8_{-2.0}$ & 55.9 & 56.0 & 53.35 \\
\hline $\log U$ & $-2.29_{-0.19}^{+0.22}$ & $-1.36_{-0.16}^{+0.17}$ & -1.29 & $-2.03-0.53$ & $-1.40-0.65$ & -1.53 & -1.41 & -2.18 \\
\hline $12+\log (\mathrm{O} / \mathrm{H})$ & $8.08_{-0.24}^{+0.51}$ & $7.86_{-0.59}^{+0.29}$ & 8.36 & $8.36_{-0.97}^{+0.24}$ & $8.34_{-0.65}^{+0.19}$ & 8.36 & 7.83 & 8.27 \\
\hline$Z / Z_{\odot}$ & $0.24_{-0.10}^{+0.56}$ & $0.15_{-0.11}^{+0.14}$ & 0.46 & $0.46_{-0.41}^{+0.36}$ & $0.45_{-0.35}^{+0.25}$ & 0.47 & 0.14 & 0.38 \\
\hline $\log (\mathrm{C} / \mathrm{O})$ & $<-0.40$ & $<-1.0$ & -0.54 & $<-1.19$ & $<-1.12$ & -0.18 & -0.54 & -0.93 \\
\hline $\log (\mathrm{N} / \mathrm{O})$ & $0.03_{-0.08}^{+0.20}$ & $0.28_{-0.24}^{+0.44}$ & -1.39 & $-0.51_{-0.63}^{+0.05}$ & $0.14_{-0.44}^{+0.15}$ & -1.24 & -1.24 & -1.39 \\
\hline
\end{tabular}

\section{DISCUSSION}

\subsection{Ly $\alpha$ and HeII emission}

The Ly $\alpha$ and HeII fluxes play an important role in characterizing the SED of the embedded ionizing source of LAEs (e.g. Sobral et al. 2015). In fact, the lack of HeII emission in the majority of the LAE spectra indicates a drop in emission at about $54.4 \mathrm{eV}$, while high Ly $\alpha$ luminosity requires high $Q(\mathrm{H})$ values. Therefore, the comparison between Ly $\alpha$ and HeII fluxes in LAEs with those observed in objects with known nature or with photoionization model predictions, is the first step to uncover the nature of the LAE ionizing source. In the bottom panel of Fig. 5, we see that the AGN observational data (see Sect. 2.2) form a clear linear trend, which can be represented by

$\log [L(\mathrm{Ly} \alpha)]=0.96( \pm 0.05) \log [L($ HeII $)]+2.56( \pm 2.23)$

with the high- $z$ radio galaxies occupying the upper end of this sequence. It can be noted that the Ly $\alpha$ luminosity of the LAEs is more consistent with the one of quasars and of less bright high- $z$ radio galaxies, and they are brighter than Seyfert 2 by a factor of $\sim 100$.

Let us define the ratio

$R=\frac{L(\operatorname{He} \mathrm{II})}{L(\operatorname{Ly} \alpha)}$

as a feature of the field radiation. Concerning the $R$ values for different classes of AGNs from the control sample, for high- $z$ radio galaxies these are in the range 0.03-0.60, for Seyfert 2 in the range 0.01-0.3, and for Quasars in the range 0.06-0.30. From Table 1, for CR7 C we derived $R=0.38$; a value consistent with the $R$ values for high- $z$ radio galaxies. For COSY we obtained $R=0.05$, consistent with the $R$ values of all classes of AGNs. For other LAEs only the upper limit for HeII was defined, therefore the $R$ ratios were not calculated.

Now, we compare $R$ values predicted by the models with the ones of the LAEs. The AGN 
and DCBH models predict $R$ values in the range 0.002-0.11 and 0.006-0.012, respectively, while SF models estimate 0.0001-0.03. Thus, only AGN models reproduce the $R$ value of COSY (0.05) and all models fail to reproduce that for CR7 C (0.38). The discrepancy found for CR7 C could be due to Ly $\alpha$ escape from the nebulae, alternative Ly $\alpha$ production mechanisms, dust attenuation (Matthee et al. 2017), resonant scattering of Ly $\alpha$, and/or the presence of PopIII stellar cluster as a secondary ionizing source.

\subsection{LAE properties}

\subsubsection{ID3}

Mainali et al. (2017) found that the ionizing source of ID3 is probably a low metallicity stellar population. This result is mainly derived from comparison between the ID3 line ratio intensities HeII/CIv versus OIII]/CIv; and CIv/HeII versus OIII]/HeII with observational data and photoionization predictions. Based on the empirical and theoretical diagrams C43 $=\log [(\mathrm{CIV} \lambda 1549+\mathrm{CIII}] \lambda 1909) /$ HeII $\lambda 1640]$ versus CIII $] /$ CIV; and CIV/Ly $\alpha$ versus OIII]/Ly $\alpha$, we confirm the result found by Mainali et al. (2017) where the main ionizing source of ID3 is a young stellar cluster. Since the results above are derived using an upper limit for HeII $\lambda 1640$, these are somewhat uncertain and deeper spectroscopic data would be needed to unveil the true nature of this object.

From the detailed modelling procedure in Sect. 4.2, we derived the number of ionizing photons $\log Q(\mathrm{H}) \approx 56$ which, taking into account the STARBURST99 (Leitherer et al. 1999) predictions for instantaneous star formation, indicates the presence of ionizing stellar cluster(s) with mass of $\sim 10^{9} M_{\odot}$. The metallicity derived for this object is $\left(Z / Z_{\odot}\right)=0.46$, with $\log (\mathrm{N} / \mathrm{O})=-1.39$ and $\log (\mathrm{C} / \mathrm{O})=-0.54$. Mainali et al. (2017) did not estimate these specific abundances for this object, but reported that ID3 has a low gas metallicity. In Fig. 9 (bottom panel), we compared our ID3 predictions for $\log (\mathrm{C} / \mathrm{O})$ vs. $12+\log (\mathrm{O} / \mathrm{H})$ with abundance estimates for local star-forming regions $(z<0.1)$ and for the star forming galaxy Q2343-BX418 ( $z \sim 2.3)$, calculated from direct measurements of the electron temperature. We can see that the estimates for ID3 are consistent with the ones for SFs. Concerning the N/O abundance ratio, in Fig. 9] (top panel), our predictions are compared with those of local SFs as well as with estimates for Seyfert 2 AGNs, derived from photoionization models by Dors et al. (2017a). Again, since it was not possible to fit the Nv/HeII ratio for ID3, this object is not represented in the $\mathrm{N} / \mathrm{O}-\mathrm{O} / \mathrm{H}$ diagram. The agreement between element 
abundance estimates of ID3 and those seen for SFs additionally suggests that ID3 is a star forming galaxy.

\subsubsection{COSY}

Laporte et al. (2017) compared the observational emission line ratios CirI]/HeII versus Nv/HeII, and versus Nv/CIV of this object with those predicted by AGN and SF photoionization models. These authors found that COSY data is irreconcilable with SF models and due to the high value of $\mathrm{NV}$ emission line, the data is located at the extreme end of the AGN model predictions. As can be seen in Figure 7 of Laporte et al. (2017), the photoionization models considered by these authors indicate that COSY has a logarithm of the ionization parameter higher than -0.5 and metallicity $\left(Z / Z_{\odot}\right)>0.5$. From our analysis using all diagrams where it was possible to include COSY data, we obtain a non-thermal (AGN/DCBH) ionizing source for COSY in agreement with Laporte et al. (2017).

From the high value of the intensity of the Nv/HeII ratio, we derived a high N/O abundance ratio for this object from our detailed modelling. In Fig. 9, the $\log (\mathrm{C} / \mathrm{O})$ and $\log (\mathrm{N} / \mathrm{O})$ versus $\log (\mathrm{O} / \mathrm{H})$ values obtained for COSY (see Table 4) are compared with those obtained in

SFs and AGNs. Since only the upper limit of the carbon lines were quoted by Laporte et al. (2017), it was only possible to derive the upper limit for carbon abundance. We can see in Fig. 9 that the $\mathrm{C} / \mathrm{O}$ value obtained by the COSY AGN-model follows the tendency derived for SFs, however, the value derived by the DCBH-model is out of the sequence. Unfortunately, carbon abundance determinations for AGNs are rare in the literature. Concerning N/O, both AGN and DCBH model predictions are higher by $\sim 0.6$ dex than those of SFs with similar oxygen abundances. The N/O values derived for COSY are higher than those derived for local Seyfert 2 nuclei by Dors et al. (2017a). It is worth noting that Dors et al. (2017a) pointed out that N/O and O/H abundances in Seyfert 2 are similar to those derived for local extragalactic disc HiI regions with high metallicity. However, this result is not valid when the $\mathrm{N} / \mathrm{O}-\mathrm{O} / \mathrm{H}$ estimations for COSY are considered.

\subsubsection{CR' clump C}

Concerning CR7 C, Sobral et al. (2017) compared photoionization model predictions with observed line ratios and concluded that it may host a high ionization AGN, with low metallicity $\left(\sim 0.05-0.2 Z_{\odot}\right)$ and high ionisation parameter $(\log U \approx-2.5)$. Sobral et al. (2017) 
pointed out that the results above were obtained if the barely measured Nv line is emitted by the clump C. Matthee et al. (2017) presented spectroscopic follow-up observations of CR7 with ALMA and, based on [CII] $158 \mu \mathrm{m}$ emission, inferred the same range of metallicity above. However, a very low metallicity $\left(\approx 0.005 Z_{\odot}\right)$ was derived by Bowler et al. (2017).

Our analysis indicates that CR7 clump $\mathrm{C}$ hosts an AGN/DCBH, such as suggested by Sobral et al. (2017). In Fig. 9 (bottom panel), it can be seen that our C/O abundance results for this object is significantly lower than the one derived for other objects with similar metallicity. This is due to the relative low limit for the ratio CIV/HeII. The N/O-O/H values derived from AGN-models are in consonance with those derived for local Seyfert 2, however, the DCBH-model predicts a higher $\mathrm{N} / \mathrm{O}$ value. This is in fact also noted in COSY and is due to the softer SED of DCBH as compared to the AGN (see Fig. 4), which results in lower NV line intensities than AGN models with the same abundances. Thus, in the DCBH detailed models we must assume higher nitrogen abundance and ionization parameters (see Table 4) than the ones considered in the AGN models.

\subsubsection{J233408}

Shibuya et al. (2018) found that the constraints on the line ratios Oiı] $\lambda 1663 / \mathrm{CIV} \lambda 1549$ versus HeII $\lambda 1640 /$ CIV $\lambda 1549$ of J233408 are compatible with the predictions of SF models of Feltre et al. (2016). Our classification, based on only the diagram CIV/Ly $\alpha$ versus OIII]/Ly $\alpha$, indicates that J233408 hosts an AGN/DCBH. However, due to the few number of objects in the control sample (see Fig. 6) and the problem related to Ly $\alpha$ leakage/scattering, our LAE classification could be rather uncertain.

The metallicity derived from the detailed modelling was $Z / Z_{\odot}=0.46(0.14)$ for $\mathrm{AGN}$ (DCBH) models, respectively. Until now, these are the only metallicity estimates available in literature for J233408. The detailed modelling was based on the fitting of the CIV/HeII ratio, which makes the abundance results somewhat uncertain. In Fig. 9 (bottom panel), it can be seen that the $\mathrm{C} / \mathrm{O}$ abundance for J233408 derived in AGN and DCBH models follows the trends derived for the SFs. Since neither measurements nor limits for the Nv lines are available, the $\mathrm{N} / \mathrm{O}$ value in the models was considered to be solar and we refrain from representing it in Fig. 9 (upper panel). 


\subsubsection{A1703-zd6}

Stark et al. (2015) demonstrated that the spectral properties of A1703-zd6 can be reproduced by photoionization models of a young ionizing source which is very hot and metal poor, as well as an AGN. In both cases these authors found a very low gas metallicity, lower than $Z / Z_{\odot} \approx 0.02$, with $\log U=-1.35_{-0.40}^{+0.24}$ for the SF models. Considering the diagnostic diagrams in Sect. 4, we also found that A1703-zd6 can be classified as SF. Our detailed models indicate a higher metallicity $\left(Z / Z_{\odot} \approx 0.4\right)$ for this object than the one derived by Stark et al. (2015), but similar $U$ value. The discrepancy between the $Z$ values above is probably due to the $\mathrm{C} / \mathrm{O}$ abundance assumed in the models rather than the use of different SEDs (Stark et al. 2015 did not explicitly state the C/O value used). In Fig. 9, we can see that the $\mathrm{C} / \mathrm{O}$ versus $\mathrm{O} / \mathrm{H}$ predictions indicate a lower $\mathrm{C} / \mathrm{O}$ abundance than the one derived for objects with similar metallicity. Again, since Nv line intensity data is not available, the $\mathrm{N} / \mathrm{O}$ value in the models was considered and it is not represented in Fig. 9 (upper panel).

\section{CONCLUSIONS}

We compared the available observational ultraviolet emission line intensities of five LAEs located at high redshift $(5.7<z<7.2)$ with predictions from photoionization models built with an ionizing source representative of an AGN, a Direct Collapse Black Hole (DCBH), and stellar clusters of Population II stars. From our analysis, we conclude the following.

(i) Based on diagnostic diagrams, we concluded that CR7 (clump C), HSC J233408+004403 and COSY probably have a non-thermal ionizing source (AGN or DCBH). RXC J2248.74431-ID3 and A1703-zd6 seem to have a stellar cluster as ionizing source. It must be noted that due to the few number and the low significance of the detected emission lines the classification above could be rather uncertain.

(ii) Detailed photoionization model fittings indicate a metallicity range of $0.1 \lesssim\left(Z / Z_{\odot}\right) \lesssim$ 0.5 for the LAEs considered.

(iii) The $\mathrm{C} / \mathrm{O}$ estimations for the LAE sample, in general, are consistent with those for local star forming objects.

(iv) In most cases, an overabundance of $\mathrm{N} / \mathrm{O}$ was derived for LAEs in relation to AGNs and SFs with similar metallicities. 


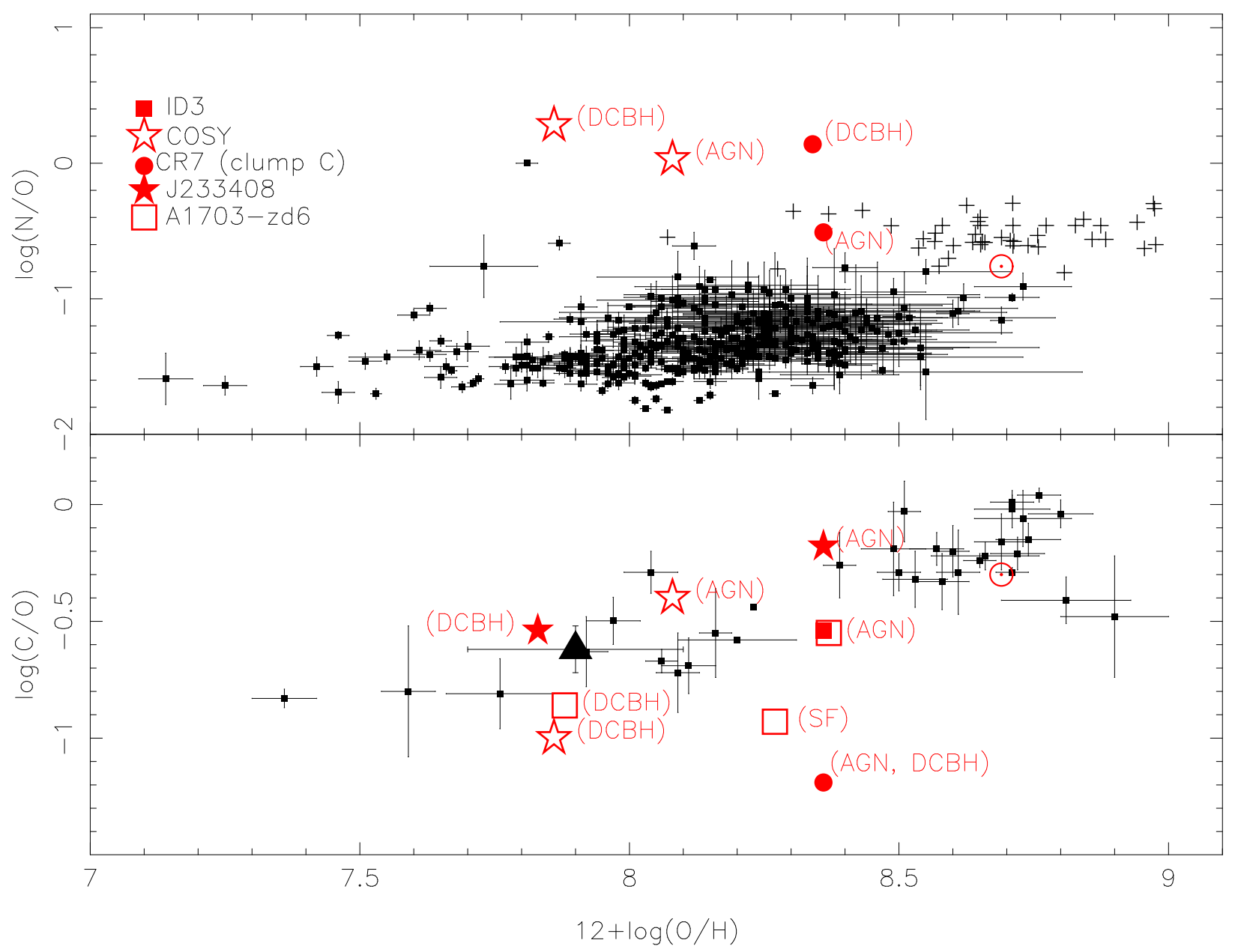

Figure 9. Bottom: $\log (\mathrm{C} / \mathrm{O})$ versus $12+\log (\mathrm{O} / \mathrm{H})$. Abundance ratios predicted by the models for the LAE sample, listed in Table 4 are indicated in the plots. The AGN, DCBH and/or SF model predictions were obtained for a given LAE (see Table (4), these are indicated in the plot. Squares represent estimations from optical-emission lines of star-forming regions by Esteban et al. 2002, 2004, 2005, 2009), Kobulnicky \& Skillman (1998) and Garnett et al. (1995, 1997). Triangle represents the estimation for the galaxy Q2343-BX418 $(z \approx 2.3)$ by Erb et al. (2010). The solar symbols (in red) represent the values for solar abundance taken from Allende Prieto et al. (2001, 2002) and Holweger (2001). Top: $\log (\mathrm{N} / \mathrm{O})$ versus $12+\log (\mathrm{O} / \mathrm{H})$. Black symbols are as in bottom panel but data were taken from Hägele et al. (2008, 2011, 2012), Pérez-Montero et al. (2011), Guseva et al. (2011), Esteban et al. (2009), Kobulnicky \& Skillman (1998), and Izotov et al. (2006). Crosses are estimations for local Seyfert 2 AGNs $(z<0.1)$ based on photoionization models performed by Dors et al. (2017a). Only the N/O and $\mathrm{O} / \mathrm{H}$ predictions for the LAEs in which was possible estimate the value for the line ratio Nv/HeII (i.e. COSY and CR7 (C)) are represented in this diagram. The error in our abundance estimations are about 0.2 dex (see Sect. 4.2).

\section{REFERENCES}

Agarwal B. et al., 2013, MNRAS, 432, 3438

Agarwal B. et al., 2017, MNRAS, 469, 231

Allen M. G., Dopita M. A., Tsvetanov Z., 1998, ApJ, 493, 571

Alende Prieto, C., Lambert, D. L., Asplund, M. 2001, ApJ, 556, L63

Alende Prieto, C., Lambert, D. L., Asplund, M. 2002, ApJ, 573, L137

Baldwin J. A., Phillips M. M., Terlevich R., 1981, PASP, 93, 5

Bertelli G., Bressan A., Chiosi C., Fagotto F.. Nasi E., 1994, A\&AS, 106, 275

Berg D. A., Skillman E. D., Henry R. B. C., Erb D. K., Carigi L., 2016, ApJ, 827, 126 
Bergeron J., Maccacaro T., Perola C., 1981, A\&A, 97, 94

Bowler R. A. A., McLure, R. J., Dunlop, J. S., et al. 2017, MNRAS, 469, 448

Bresolin F., Kennicutt R. C., Garnett D. R. 1999, ApJ, 510, 104

Bradley L. D. et al., 2012, ApJ, 747, 3

Bromm V., \& Loeb A., 2003, ApJ, 596, 34

Castro C. S., Dors O. L., Cardaci M. V., Hägele G. F., MNRAS, 467, 1507

Copetti M. V. F., Pastoriza M. G., Dottori H. A. 1985, A\&A, 152, 427

Copetti M. V. F., Castanẽda H. O., Mallmann J. A. H., Schmidt A. A., 2000, A\&A, 357, 621

Contini M., 2017, MNRAS, 469, 3125

Colina L., 2015, A\&A, 578, 48

de Barros S. et al., 2016, A\&A, 585, 51

De Robertis M. M., Hutchings J. B., Pitts R. E., 1988, AJ, 95, 1371

Díaz, Á. I., Terlevich, E., Castellanos, M., \& Hägele, G. F. 2007, MNRAS, 382, 251

Dopita M. A. et al., 2015, ApJS, 217, 12

Dopita M. A. et al., 2006, ApJSS, 167, 177

Dors O. L., Arellano-Córdova K. Z., Cardaci M. V., Hägele G. F., 2017a, MNRAS, 468, L113

Dors O. L., Hägele G. F., Cardaci M. V., Krabbe A. C., 2017b, MNRAS, 466, 726

Dors O. L. et al., 2015, MNRAS, 453, 4102

Dors O. L., \& Copetti M. V. F., 2006, A\&A, 452, 437

Dors O. L., Cardaci M. V., Hägele G. F., Krabbe A. C., 2014, MNRAS, 443, 1291

Dors O. L., Krabbe A. C, Hägele G. F., Pérez-Montero E., 2011, MNRAS, 415, 3616

Erb D. K. et al., 2010, ApJ, 719, 1168

Esteban C., Bresolin, F., Peimbert, M. et al. 2009, ApJ, 700, 654

Esteban C., García-Rojas, J., Peimbert, M. et al. 2005, ApJ, 618, L95

Esteban C., Peimbert, M., García-Rojas, J., et al. 2004, MNRAS, 355, 229

Esteban C., Peimbert, M., Torres-Peimbert, S., Rodrguez, M. 2002, ApJ, 581, 241

Feltre A., Charlot S., Gutkin J., 2016, MNRAS, 456, 3354

Ferland G. J., 2013, Rev. Mex. Astron. Astrofis., 49, 137

Garnett D. R., Shields, G. A., Skillman, E. D., Sagan, S. P., Dufour, R. J. 1997, ApJ, 489, 63

Garnett D. R., Skillman, E. D., Dufour, R. J. et al. 1995, ApJ, 443, 64 
Groves B. A., Heckman T. M., Kauffmann G., 2006, MNRAS, 371, 1559

Grazian A. et al., arXiv:1802.01953

Guseva N. G., Izotov, Y. I., Stasińska, G. et al. 2011, A\&A, 529, 149

Hägele G. F., Firpo, V., Bosch, G., Díaz, Á. I., \& Morrell, N. 2012, MNRAS, 422, 3475

Hägele G. F., García-Benito, R., Pérez-Montero, E., et al. 2011, MNRAS, 414, 272

Hägele G. F. et al. 2008, MNRAS, 383, 209

Hägele G. F., Pérez-Montero, E., Díaz, Á. I., Terlevich, E., \& Terlevich, R. 2006, MNRAS, 372,293

Hayes M., 2015, PASA, 32, 27

Holweger H., 2001, AIP Conference Proceedings, Vol. 598, pp.23-30

Izotov, Y. I., Stasińska, G., Meynet, G., Guseva, N. G., Thuan, T. X. 2006, A\&A, 448, 955

Jaffe W. et al., 2004, Nature, 429, 47

Kennicutt R. C., Bresolin, F., Garnett D. R. 2003, ApJ, 591, 80

Kewley L. J., \& Ellison S. L., 2008, ApJ, 681, 1183

Krabbe A. C. et al., 2014, MNRAS, 437, 1155

Kobulnicky H. A., Skillman, E. D., 1998, ApJ, 497, 601

Koski A. T., 1978, ApJ, 223, 56

Latif M. A., Omukai K., Habouzit M., Schleicher D. R. G., Volonteri M., 2016, ApJ, 823, 40

Laporte N. et al., 2017, ApJ, 851, 40

Leclercq F. et al., 2017, A\&A, 608, 8

Leitherer C. et al., 1999, ApJ, 123, 3

Lehnert M. D. et al., 2010, Nature, 467, 940

Levesque E. M., Leitherer C., Ekstrom C., Meynet G., Schaerer D., 2012, ApJ, 751, 67

López-Gonzaga N., Burtscher L., Tristram K. R. W., Meisenheimer K., Schartmann M., 2016, A\&A, 591, 47

Madau P., \& Haardt F., 2015, ApJ, 813, L8

Mainali R., Kollmeier J. A., Stark D. P et al., 2017, ApJ, 836,14L

Matthee J. et al., 2017, ApJ, 851, 145

Matthee J. et al., 2015, MNRAS, 451, 400

Miller B. P., Brandt W. N., Schneider D. P., Gibson R. R., Steffen A. T., Wu J., 2011, ApJ, 726, 20

Nakajima K. et al., 2018, arxvi:1801.03085 
Nakajima K. et al., 2017, arxvi:1709.03990

Oesch P. A. et al., 2015, ApJL, 804, L30

Omukai K., Schneider R., Haiman Z., 2008, ApJ, 686, 801

Ouchi M. et al., 2009, ApJ, 696, 1164

Ouchi M. et al., 2008, ApJS, 176, 301

Ota K. et al., 2008, ApJ, 677, 12

Pallottini A. et al., 2015, MNRAS, 453, 2465

Pacucci F., Pallottini A., Ferrara A., Gallerani S., 2017, MNRAS, 468, 77L

Partridge R. B., \& Peebles P. J. E., 1967, ApJ, 147, 868

Pauldrach A. W. A., Hoffmann T. L., Lennon M., 2001, A\&A, 375, 161

Pérez-Montero E, \& Amorín R., 2017, MNRAS, 467, 1287

Pérez-Montero E., Vílchez, J. M., Cedrés, B. et al. 2011, A\&A, 532, 141

Pritchet C. J., 1994, PASP, 106, 1052

Reunanen J., Kotilainen J. K., Prieto M. A., 2002, MNRAS, 331, 154

Revalski M. et al., 2018, arXiv:1802.07734

Riffel R. et al., 2013, MNRAS, 432, 3545

Roberts-Borsani G. W., Bouwens R. J., Oesh P. A. et al., 2016, ApJ, 823, 143

Rodríguez-Ardila A., Pastoriza M. G., Viegas S., Sigut T. A. A., Pradhan A. K., A\&A, 425,457

Rivera-Thorsen T. E. et al., 2017, A\&A, 608, 4L

Rydberg C.-E. et al., 2015, ApJ, 804, 13

Rydberg C.-E. et al., 2017, MNRAS, 467, 768

Sanders R. L. et al., 2016, ApJ, 816, 23

Schmidt K. B., Huang K. -H., Treu T. et al. 2017, ApJ, 839, 17

Shakura N. I., \& Sunyaev R. A., 1973, A\&A, 24, 337

Schenker M. A. et al., 2012, ApJ, 744, 179

Senchyna P. et al., 2017, MNRAS, 472, 2608

Shibuya T. et al. 2018, PASJ, 70, 15

Sobral D. et al., 2018, MNRAS, 477, 2817

Sobral D. et al., 2017, arXiv:1710.08422

Sobral D. et al., 2015, ApJ, 808, 139

Stasinska G., \& Izotov I., 2003, A\&A, 397, 71

Stark D. P. et al., 2017, MNRAS, 464, 469 
Stark D. P. et al., 2015, MNRAS, 454, 1393

Stark D. P. et al., 2014, MNRAS, 445, 3200

Stark D. P. et al., 2010, MNRAS, 408, 1628

Steidel C. C. et al., 2016, ApJ, 826, 159

Thomas A. D., 2018, arXiv:180300740

Thuan T. X., 1984, ApJ, 281, 126

van Hoof P. A. M. Photo-ionization studies of nebulae. PhD thesis, Rijksuniversiteit Groningen, 1997

Vanzella E., et al., 2016, ApJL, 821, L27

Villar-Martín M., Cerviño M., González Delgado R. M., 2004, MNRAS, 355, 1132

Vila-Costas M. B., \& Edmunds M. G. 1993, MNRAS, 265, 199

Visbal E., Haiman Z., Bryan G. L., 2016, MNRAS, 460, L59

Zabl, J.; Norgaard-Nielsen, H. U.; Fynbo, J. P. U.; Laursen, P.; Ouchi, M.; Kjrgaard, P., 2015, MNRAS, 451, 2050

Zamorani G. et al., 1981, ApJ, 245, 357

Zastrow J., Oey M. S., Pellegrini E. W., 2013, ApJ, 769, 94

Zheng W. et al., 2009, ApJ, 697, 1907

Wright, E. L. 2006, PASP, 118, 1711 